\title{
Weak decays of $\bar{B}_{S}$ mesons
}

\author{
C. Albertus \\ Departamento de Física Atómica, Molecular y Nuclear. Universidad de Granada. \\ Avenida de Fuentenueva S/N, E-18071 Granada, Spain
}

In the present work we study the semileptonic decays of $\bar{B}_{S}$ mesons in the context of nonrelativistic constituent quark models. We estimate the uncertainties of our calculation using different interquark potentials to obtain the meson wave functions. We check the results from our model against the predictions of Heavy Quark Symmetry, in the limit of infinite heavy quark mass. We also study the nonleptonic decays of $\bar{B}_{s}$ mesons within the factorization approximation. 


\section{INTRODUCTION}

Since the first claims on the existence of $B_{S}$ and $\bar{B}_{s}$, both their lifetimes, decay modes [1-12] and oscillations [13-19] have been objectives of the uttermost interest of experimental collaborations. Being below the $B-K$ threshold, it can only decay by means of mechanisms governed by electroweak currents, making it an ideal system to study the physics of the weak interaction in the presence of heavy quarks.

A considerable amount of the work devoted to the $b$-meson sector involve the ideas of Heavy Quark Symmetry [20, 21] (HQS). HQS is an approximate symmetry of QCD that becomes exact in the limit in which the mass of the heavy quark becomes infinity. This symmetry establishes that in such a limit, the quantum numbers of the light degrees of freedom are all well defined, and independent of the heavy quark flavor and spin. This is similar, for instance, to what happens in atomic physics, where electron properties are approximately independent of the mass and spin of the nucleus for a fixed nuclear charge. Heavy Quark Symmetry can be cast into the language of an effective theory, leading to Heavy Quark Effective Theory [22] (HQET). HQET enables a systematic, order by order evaluation of the corrections to the infinity mass limit in the inverse powers of the heavy quark masses. Besides, HQET allows theoretical control of the non-perturbative aspects of the calculation in the proximities of the infinite quark mass limit. At leading order in an expansion on the heavy quark mass only one form factor, the Isgur-Wise function remains, largely simplifying the description of the decay. However, HQS does not determine the Isgur-Wise function: one still needs to implement some other nonperturbative method.

HQS leads to many more model independent predictions. The most remarkable of those for the meson sector, is the fact that the masses of pseudoscalar and vector mesons are degenerate in the heavy quark limit. Nonrelativistic quark models fulfil this constrain: the reduced mass of the two quarks is just the mass of the light one, and the spin-spin terms, which can distinguish vector from pseudoscalar, are suppressed by the mass of the heavy quark, becoming exactly zero in the HQS limit. At this point, one important question is to what extent do the deviations from the HQS limit, evaluated from nonrelativistic quark models agree with the constraints predicted by HQET. Furthermore, it is possible to make use of the HQET constrains to improve the predictions of the quark models. In the previous work of Ref. [23], we studied the leptonic and semileptonic decays of $B$ mesons, and considered the implications of HQS. The nonleptonic and semileptonic decay of $B_{c}$ mesons (where presence of two heavy quarks leads to infrared divergences that break the flavor symmetry, and subsequently only Heavy Quark Spin Symmetry remains), has been considered in Ref. [24]. In Refs. [25-27] we calculated the semileptonic decay widths of baryons containing one or two heavy quarks, and worked out the symmetry implications on the observables.

Some of the decay modes of $B_{s}$ or $\bar{B}_{s}$ mesons have been studied within the framework of relativistic constituent quark model [28, 29], perturbative QCD [30, 31], Bethe-Salpeter techniques [32], light front quark model [33], sum rules [34-36] or non relativistic constituent quark model [37] for instance. In this paper we study the semileptonic and nonleptonic decay of $\bar{B}_{s}$ in the context of nonrelativistic constituent quark model. The rest of the paper is organized as follows. In Section $\Pi$ we describe the meson states for the different values of $J^{P}$ and the quark models used in this work. In Sec. [II] we give the form factor decomposition of the weak decay matrix elements and calculate the decay width, both for light $(e, \mu)$ and heavy $(\tau)$ charged lepton. We also work in the helicity formalism [38]. Besides, we study the implications of HQS in these decays. In Sec. V the problem of nonleptonic two meson decays of $B_{s}$ is studied. The meson decay constants required in Sec. $\nabla$ and the CKM matrix elements used both in Secs. [II and $\mathrm{V}$ can be found in Tables $\Pi$ respectively, while the different $D_{s}$ states considered in the semileptonic and some nonleptonic decays studied in this paper and their quantum numbers are summarized in Table $\Pi$. For the $D_{s}^{1}(2460)$ and $D_{s}^{1}(2536)$ states, we assume that they are mixing of ${ }^{3} P_{1}$ and ${ }^{1} P_{1} c \bar{s}$ states, with a mixing angle of $34.5^{\circ}$, as in Ref. [28]. In Sec.VII] we present a summary and our conclusions. The paper also includes an Appendix to clarify some technical details of our work.

\begin{tabular}{cc}
\hline \hline & Mass $(\mathrm{MeV})$ \\
\hline $\bar{B}_{s}\left(0^{-}\right)$ & $5366.77[39]$ \\
$D_{s}^{+}\left(0^{-}\right)$ & $1968.49[39]$ \\
$D_{s 0}^{*+}(2317)\left(0^{+}\right)$ & $2317.8[39]$ \\
$D_{s}^{*+}\left(1^{-}\right)$ & $2112.3[39]$ \\
$D_{s 1}(2460)$ & $2459.6[39]$ \\
$D_{s 1}(2536)$ & $2535.12[39]$ \\
$c \bar{s}\left(2^{-}\right)$ & 2806.9 \\
$D_{s 2}(2573)^{+}\left(2^{+}\right)$ & $2571.9[39]$ \\
\hline
\end{tabular}

TABLE I. Masses of the states involved in this calculation. 


\section{MESON STATES AND INTERQUARK INTERACTIONS}

In the context of nonrelativistic constituent quark models, the state of a meson $M$ is written as [40]:

$$
\begin{aligned}
|M ; \lambda \vec{P}\rangle_{\mathrm{NR}}= & \int d^{3} p \sum_{\alpha_{1} \alpha_{2}} \hat{\phi}_{\alpha_{1} \alpha_{2}}^{(M, \lambda)}(\vec{p}) \frac{(-1)^{(1 / 2)-s_{2}}}{(2 \pi)^{3 / 2} \sqrt{\left(2 E_{f_{1}}\left(\vec{p}_{1}\right)\right)\left(2 E_{f_{2}}\left(\vec{p}_{2}\right)\right)}} \\
& \times\left|q, \alpha_{1} \vec{p}_{1}=\frac{m_{f_{1}}}{m_{f_{1}}+m_{f_{2}}} \vec{P}-\vec{p}\right\rangle\left|\vec{q}, \alpha_{2} \vec{p}_{2}=\frac{m_{f_{2}}}{m_{f_{1}}+m_{f_{2}}} \vec{P}+\vec{p}\right\rangle,
\end{aligned}
$$

where $\vec{P}$ is the meson three momentum, while $\lambda$ labels the spin projection in the meson center of mass. The index $\alpha_{i}$ represent the quantum numbers of spin, flavor and color of the quark and the antiquark, with four momentum and mass given by $\left(E_{f_{i}}\left(\vec{p}_{i}\right), \vec{p}_{i}\right)$ and $m_{f_{i}}$ respectively. The factor $(-1)^{1 / 2-s_{2}}$ ensures that the antiquark spin states have the correct phase 1 .

The normalization of quark and antiquark states is

$$
\left\langle\alpha^{\prime} \vec{p}^{\prime} \mid \alpha \vec{p}\right\rangle=2 E_{f} \delta_{\alpha^{\prime} \alpha} \delta^{3}\left(\vec{p}-\vec{p}^{\prime}\right)(2 \pi)^{3}
$$

As for the momentum wave function accounting for the relative motion of the quark-antiquark system, the normalization is given by

$$
\int d^{3} p \sum_{\alpha_{1} \alpha_{2}}\left(\hat{\phi}_{\alpha_{1} \alpha_{2}}^{M, \lambda^{\prime}}(\vec{p})\right)^{*} \hat{\phi}_{\alpha_{1} \alpha_{2}}^{M, \lambda}(\vec{p})=\delta_{\lambda \lambda^{\prime}}
$$

and finally, the normalization of the meson states in our model is

$$
\mathrm{NR}\left\langle M \lambda^{\prime} \vec{P}^{\prime} \mid M \lambda \vec{P}\right\rangle_{\mathrm{NR}}=\delta_{\lambda \lambda^{\prime}}(2 \pi)^{3} \delta\left(\vec{P}^{\prime}-\vec{P}\right) .
$$

In this calculation we will need the ground state wave function for scalar $\left(0^{+}\right)$, pseudoscalar $\left(0^{-}\right)$, vector $\left(1^{-}\right)$, axial-vector $\left(1^{+}\right)$, tensor $\left(2^{+}\right)$and pseudotensor $\left(2^{-}\right)$. Assuming always a value for the orbital angular momentum as low as possible, we have for a meson $M$ with scalar, pseudoscalar and vector quantum numbers

$$
\begin{aligned}
\hat{\phi}_{\alpha_{1}, \alpha_{2}}^{\left(M\left(0^{+}\right)\right)}(\vec{p}) & =\frac{1}{\sqrt{3}} \delta_{c_{1} c_{2}} \hat{\phi}_{\left(s_{1}, f_{1}\right),\left(s_{2}, f_{2}\right)}^{\left(M\left(0^{+}\right)\right.}(\vec{p})=\frac{i}{\sqrt{3}} \delta_{c_{1} c_{2}} \hat{\phi}_{f_{1}, f_{2}}^{\left(M\left(0^{+}\right)\right)}(|\vec{p}|) \sum_{m}\left(1 / 2,1 / 2,1 ; s_{1}, s_{2},-m\right)(1,1,0 ; m,-m, 0) Y_{1 m}(\hat{p}) \\
\hat{\phi}_{\alpha_{1}, \alpha_{2}}^{\left(M\left(0^{-}\right)\right)}(\vec{p}) & =\frac{1}{\sqrt{3}} \delta_{c_{1} c_{2}} \hat{\phi}_{\left(s_{1}, f_{1}\right),\left(s_{2}, f_{2}\right)}^{\left(M\left(0^{-}\right)\right)}(\vec{p})=\frac{-i}{\sqrt{3}} \delta_{c_{1} c_{2}} \hat{\phi}_{f_{1}, f_{2}}^{\left(M\left(0^{-}\right)\right)}(|\vec{p}|)\left(1 / 2,1 / 2,0 ; s_{1}, s_{2}, 0\right) Y_{00}(\hat{p}) \\
\hat{\phi}_{\alpha_{1}, \alpha_{2}}^{\left(M\left(1^{-}\right), \lambda\right)}(\vec{p}) & =\frac{1}{\sqrt{3}} \delta_{c_{1} c_{2}} \hat{\phi}_{\left(s_{1}, f_{1}\right),\left(s_{2}, f_{2}\right)}^{\left(M\left(1^{-}\right), \lambda\right)}(\vec{p})=\frac{-1}{\sqrt{3}} \delta_{c_{1} c_{2}} \hat{\phi}_{f_{1}, f_{2}}^{\left(M\left(1^{-}\right)\right)}(|\vec{p}|)\left(1 / 2,1 / 2,1 ; s_{1}, s_{2}, 0\right) Y_{00}(\hat{p}),
\end{aligned}
$$

where $\left(j_{1}, j_{2}, j_{3}, m_{1}, m_{2}, m_{3}\right)$ are Clebsch-Gordan coefficients, $Y_{l m}$ are spherical harmonics and $\hat{\phi}_{f_{1}, f_{2}}(|\vec{p}|)$ is the Fourier transform of the radial, coordinate space, wave function.

Axial vector mesons require orbital angular momentum $L=1$, and in this case the two possible values of the total quarkantiquark spin $S_{q \bar{q}}=0,1$ are allowed. Thus, there are two possible states:

$$
\begin{aligned}
\hat{\phi}_{\alpha_{1}, \alpha_{2}}^{\left(M\left(1^{+}\right), S_{q \bar{q}}=0, \lambda\right)}(\vec{p}) & =\frac{1}{\sqrt{3}} \delta_{c_{1} c_{2}} \hat{\phi}_{\left(s_{1}, f_{1}\right),\left(s_{2}, f_{2}\right)}^{\left(M\left(1^{+}\right), S_{\bar{q}}=0, \lambda\right)}(\vec{p})=\frac{-1}{\sqrt{3}} \delta_{c_{1} c_{2}} \hat{\phi}_{f_{1}, f_{2}}^{\left(M\left(1^{+}\right), S_{q \bar{q}}=0\right)}(|\vec{p}|)\left(1 / 2,1 / 2,0 ; s_{1}, s_{2}, 0\right) Y_{1 \lambda}(\hat{p}) \\
\hat{\phi}_{\alpha_{1}, \alpha_{2}}^{\left(M\left(1^{+}\right), S_{q \bar{q}}=1, \lambda\right)}(\vec{p}) & =\frac{1}{\sqrt{3}} \delta_{c_{1} c_{2}} \hat{\phi}_{\left(s_{1}, f_{1}\right),\left(s_{2}, f_{2}\right)}^{\left(M\left(1^{+}\right), S_{\bar{q}}=1, \lambda\right)}(\vec{p}) \\
& =\frac{-1}{\sqrt{3}} \delta_{c_{1} c_{2}} \hat{\phi}_{f_{1}, f_{2}}^{\left(M\left(1^{+}\right), S_{q \bar{q}}=1\right)}(|\vec{p}|) \sum_{m}\left(1 / 2,1 / 2,1 ; s_{1}, s_{2}, \lambda-m\right)(1,1,1 ; m, \lambda-m, \lambda) Y_{1 m}(\hat{p}) .
\end{aligned}
$$

\begin{tabular}{cccccccccc||ccccc}
\hline \hline$f_{\pi}$ & $f_{\rho}$ & $f_{K}$ & $f_{K^{*}}$ & $f_{D}$ & $f_{D^{*}} \dagger$ & $f_{D_{s}}$ & $f_{D_{s}^{*}} \dagger$ & $f_{\Phi^{\dagger}}$ & $f_{J / \Psi^{\dagger}}$ & $\left|V_{c b}\right|$ & $\left|V_{u d}\right|$ & $\left|V_{u s}\right|$ & $\left|V_{c s}\right|$ & $\left|V_{c d}\right|$ \\
\hline 130.41 & 210 & 159.8 & 217 & 206.7 & 222 & 260 & 318 & 312.6 & 488.5 & 0.0413 & 0.9743 & 0.2240 & 0.9734 & 0.2252 \\
\hline
\end{tabular}

TABLE II. Values for the meson decay constants in MeV and Cabibbo-Kobayashi-Maskawa matrix elements used through this work. The decay constants marked with a † have been calculated using our model.

\footnotetext{
${ }^{1}$ Under charge conjugation $\mathrm{C}$, quark and antiquark states are related via $C c_{\alpha}^{\dagger} C^{\dagger}=(-1)^{1 / 2-s} d_{\alpha}^{\dagger}(\vec{p})$, so the antiquark states with the correct spin relative phase are $(-1)^{1 / 2-s} d_{\alpha}^{\dagger}(\vec{p})|0\rangle=(-1)^{1 / 2-s}|\bar{q}, \alpha \vec{p}\rangle$
} 
For tensor and pseudotensor mesons, the wave functions can be written as:

$$
\begin{aligned}
\hat{\phi}_{\alpha_{1}, \alpha_{2}}^{\left(M\left(D_{s 2}^{*}\right), \lambda\right)}(\vec{p}) & =\frac{1}{\sqrt{3}} \delta_{c_{1} c_{2}} \hat{\phi}_{\left(s_{1}, f_{1}\right),\left(s_{2}, f_{2}\right)}^{\left(M\left(D_{s 2}^{*}\right), \lambda\right)}(\vec{p}) \\
& =\frac{1}{\sqrt{3}} \delta_{c_{1} c_{2}} \hat{\phi}_{f_{1}, f_{2}}^{\left(M\left(D_{s 2}^{*}\right)\right)}(|\vec{p}|) \sum_{m}\left(1 / 2,1 / 2,1 ; s_{1}, s_{2}, \lambda-m\right)(1,1,2 ; m, \lambda-m, \lambda) Y_{1 m}(\hat{p}) \\
\hat{\phi}_{\alpha_{1}, \alpha_{2}}^{\left(M\left(2^{-}\right), \lambda\right)}(\vec{p}) & =\frac{1}{\sqrt{3}} \delta_{c_{1} c_{2}} \hat{\phi}_{\left(s_{1}, f_{1}\right),\left(s_{2}, f_{2}\right)}^{\left(M\left(2^{-}\right), \lambda\right)}(\vec{p}) \\
& =\frac{-1}{\sqrt{3}} \delta_{c_{1} c_{2}} \hat{\phi}_{f_{1}, f_{2}}^{\left(M\left(2^{-}\right)\right)}(|\vec{p}|) \sum_{m}\left(1 / 2,1 / 2,1 ; s_{1}, s_{2}, \lambda-m\right)(2,1,2 ; m, \lambda-m, \lambda) Y_{2 m}(\hat{p})
\end{aligned}
$$

In the previous expressions, all phases have been introduced for later convenience.

We consider five different interquark potentials to calculate the coordinate space wave functions, one proposed by Bhadury [41] and other four proposed by Silvestre-Brac in [42]. All of them have the same structure: a term accounting for confinement, plus Coulomb and hyperfine terms both of them coming from one-gluon exchange. They differ from one another in the form factors present in the hyperfine term, the power of the confinement term, or the presence of a form factor in the Coulomb one-gluon exchange term. All free parameters have been adjusted to reproduce light and heavy-light meson spectra. We have successfully used these potentials before to describe the spectra and decays of charmed and bottom baryons.

The different results obtained with the different potentials provide us with an estimation of the theoretical error. It has to be mentioned that another source of theoretical uncertainty that we cannot account for is the use of nonrelativistic kinematics in the evaluation of the wave function. While this approximation is not, a priori, a good choice in the presence of light quarks, one has to notice that all nonrelativistic potentials have free parameters fitted to experimental data. Hence, one can argue that the ignored relativistic effects are partially included in the fitted values of the parameters.

\section{SEMILEPTONIC DECAYS}

In this section we will consider the semileptonic decay of $\bar{B}_{s}$ mesons into different $D_{s}$ meson states with $0^{+}, 0^{-}, 1^{+}, 1^{-}, 2^{+}$ and $2^{-}$spin-parity quantum numbers. These decays correspond to $b \rightarrow c$ transition at the quark level governed by the current

$$
J_{\mu}^{c b}(0)=J_{V \mu}^{c b}(0)-J_{A \mu}^{c b}(0)=\bar{\Psi}_{c}(0) \gamma_{\mu}\left(I-\gamma_{5}\right) \Psi_{b}(0)
$$

with $\Psi_{f}$ a quark field with flavor $f$.

\section{A. Form factor decomposition of hadronic matrix elements}

The hadronic matrix elements involved in these processes can be parametrized in terms of form factors as:

$$
\begin{aligned}
\left\langle D_{s}^{+}, \vec{P}_{D_{s}}\left|J_{\mu}^{b c}(0)\right| \bar{B}_{s}, \vec{P}_{\bar{B}_{s}}\right\rangle & =P_{\mu} F_{+}\left(q^{2}\right)+q_{\mu} F_{-}\left(q^{2}\right) \\
\left\langle D_{s}^{*+}, \lambda \vec{P}_{D_{s}^{*}}\left|J_{\mu}^{b c}(0)\right| \bar{B}_{s}, \vec{P}_{\bar{B}_{s}}\right\rangle & =\frac{-1}{m_{\bar{B}_{s}}+m_{D_{s}^{*}}} \varepsilon_{\mu v \alpha \beta} \varepsilon_{(\lambda)}^{\nu *}\left(\vec{P}_{c \bar{s}}\right) P^{\alpha} q^{\beta} V\left(q^{2}\right) \\
& -i\left\{\left(m_{\bar{B}_{s}}-m_{D_{s}^{*}}\right) \varepsilon_{(\lambda) \mu}^{*}\left(\vec{P}_{c \bar{s}}\right) A_{0}\left(q^{2}\right)-\frac{P \cdot \varepsilon_{(\lambda)}^{*}\left(\vec{P}_{D_{s}^{*}}\right)}{m_{\bar{B}_{s}}+m_{D_{s}^{*}}}\left(P_{\mu} A_{+}\left(q^{2}\right)+q_{\mu} A-\left(q^{2}\right)\right)\right\} \\
\left\langle D_{s 2}^{*+}, \lambda \vec{P}_{D_{s 2}^{*}}\left|J_{\mu}^{b c}(0)\right| \bar{B}_{s}, \vec{P}_{\bar{B}_{s}}\right\rangle= & \varepsilon_{\mu v \alpha \beta} \varepsilon_{(\lambda)}^{v \delta *}\left(\vec{P}_{D_{s 2}^{*}}\right) P_{\delta} P^{\alpha} q^{\beta} T_{4}\left(q^{2}\right) \\
& -i\left\{\varepsilon_{(\lambda) \mu \delta}^{*}\left(\vec{P}_{D_{s 2}^{*}}\right) P^{\delta} T_{1}\left(q^{2}\right)+P^{v} P^{\delta} \varepsilon_{(\lambda) v \delta}^{*}\left(\vec{P}_{D_{s 2}^{*}}\right)\left(P_{\mu} T_{2}\left(q^{2}\right)+q_{\mu} T_{3}\left(q^{2}\right)\right)\right\}
\end{aligned}
$$

where $P_{\bar{B}_{s}}$ and $P_{c \bar{s}}$ (with $c \bar{s}=D_{s}, D_{s}^{*}, D_{s 2}^{*}$ ) are the meson four-momenta, $m_{\bar{B}_{s}}$ and $m_{c \bar{s}}$ their masses respectively, $P=P_{\bar{B}_{s}}+P_{c \bar{s}}$, $q=P_{\bar{B}_{s}}-P_{c \bar{s}} . \quad \varepsilon^{\mu v \alpha \beta}$ is the fully antisymmetric tensor, for which we have taken the convention $\varepsilon^{0123}=1$. $q^{2}$ ranges from $q_{\min }^{2}=m_{l}^{2}$ to $q_{\max }^{2}=\left(P_{\bar{B}_{s}}-P_{c \bar{s}}\right)^{2}$. It is common to use $\omega=\left(m_{\bar{B}_{s}}^{2}-m_{c \bar{s}}^{2}-q^{2}\right) / 2 m_{\bar{B}_{s}} m_{c \bar{s}}$ instead of $q^{2}$, corresponding $\omega_{\min }=1$ to 
$q_{\max }^{2} \cdot \varepsilon_{(\lambda) \mu}(\vec{P})$ and $\varepsilon_{(\lambda) \mu v}(\vec{P})$ are the polarization vector and tensor of vector and tensor mesons, respectively. The latter can be evaluated as

$$
\varepsilon_{(\lambda)}^{\mu \nu}(\vec{P})=\sum_{m}(1,1,2 ; m, \lambda-m, \lambda) \varepsilon_{(\lambda)}^{\mu}(\vec{P}) \varepsilon_{(\lambda-m)}^{v}(\vec{P}) .
$$

The different polarization vectors used in this work can be found in the Appendix of Ref. [40].

Meson states in Eq. (9) are normalized as

$$
\left\langle M, \lambda^{\prime} \vec{P}^{\prime} \mid M \lambda \vec{P}\right\rangle=\delta_{\lambda^{\prime} \lambda} 2 E_{M}(\vec{P})(2 \pi)^{3} \delta\left(\vec{P}-\vec{P}^{\prime}\right)
$$

where $E_{M}(\vec{P})$ is the energy of the meson $M$ with three momentum $\vec{P}$. The factor $2 E_{M}$ should be noticed, in contrast with Eq. (4).

For $0^{+}, 1^{+}$and $2^{-}$final states the form factor decomposition is the same as for the $0^{-}, 1^{-}$and $2^{+}$cases above, where just $-J_{A}^{c b}(0)$ is contributing instead of $J_{V}^{c b}(0)$ and vice versa.

\section{B. Decays into scalar and pseudoscalar states}

In this section we will consider the decay of $\bar{B}_{s}$ mesons into pseudoscalar and scalar $c \bar{s}$ mesons. For $\bar{B}_{s} \rightarrow D_{s}^{+}$, i. e. $\left(0^{-}\right)$ transitions, the form factors are given by

$$
\begin{aligned}
& F_{+}\left(q^{2}\right)=\frac{1}{m_{\bar{B}_{s}}}\left(V^{0}(|\vec{q}|)+\frac{V^{3}(|\vec{q}|)}{|\vec{q}|}\left(E_{D_{s}}(-\vec{q})-m_{D_{s}}\right)\right) \\
& F_{-}\left(q^{2}\right)=\frac{1}{m_{\bar{B}_{s}}}\left(V^{0}(|\vec{q}|)+\frac{V^{3}(|\vec{q}|)}{|\vec{q}|}\left(E_{D_{s}}(-\vec{q})+m_{D_{s}}\right)\right)
\end{aligned}
$$

whereas for a transition onto a $D_{s 0}^{*+}\left(0^{+}\right)$state we have

$$
\begin{aligned}
& F_{+}\left(q^{2}\right)=\frac{-1}{m_{\bar{B}_{s}}}\left(V^{0}(|\vec{q}|)+\frac{V^{3}(|\vec{q}|)}{|\vec{q}|}\left(E_{D_{s 0}^{*}}(-\vec{q})-m_{D_{s 0}^{*}}\right)\right) \\
& F_{-}\left(q^{2}\right)=\frac{-1}{m_{\bar{B}_{s}}}\left(V^{0}(|\vec{q}|)+\frac{V^{3}(|\vec{q}|)}{|\vec{q}|}\left(E_{D_{s 0}^{*}}(-\vec{q})+m_{D_{s 0}^{*}}\right)\right)
\end{aligned}
$$

where $V^{\mu}(|\vec{q}|)$ and $A^{\mu}(|\vec{q}|)(\mu=0,3)$ are calculated in our model as

$$
\begin{aligned}
& V^{\mu}(|\vec{q}|)=\left\langle D_{s}^{+},-|\vec{q}| \vec{k}\left|J_{V}^{c b \mu}(0)\right| \bar{B}_{s}, \overrightarrow{0}\right\rangle=\sqrt{4 m_{\bar{B}_{s}} E_{D_{s}}(-\vec{q})}\left\langle D_{\mathrm{NR}}^{+},-|\vec{q}| \vec{k}\left|J_{V}^{c b \mu}(0)\right| \bar{B}_{s}, \overrightarrow{0}\right\rangle_{\mathrm{NR}} \\
& A^{\mu}(|\vec{q}|)=\left\langle D_{s 0}^{*+},-|\vec{q}| \vec{k}\left|J_{A}^{c b \mu}(0)\right| \bar{B}_{s}, \overrightarrow{0}\right\rangle=\sqrt{4 m_{\bar{B}_{s}} E_{D_{s 0}^{*}}(-\vec{q})}{ }_{\mathrm{NR}}\left\langle D_{s 0}^{*+},-|\vec{q}| \vec{k}\left|J_{A}^{c b \mu}(0)\right| \bar{B}_{s}, \overrightarrow{0}\right\rangle_{\mathrm{NR}}
\end{aligned}
$$

where the expressions for the non-relativistic matrix elements are given in the Appendix. Figure 1 represents the form factors calculated with the wave functions corresponding to the AL1 potential [42].

\section{Decays into vector and axial vector states}

In the case of decays of $\bar{B}_{s}$ mesons into vector $D_{s}^{*+}$ the form factors are given by:

$$
\begin{aligned}
& V\left(q^{2}\right)=\frac{i}{\sqrt{2}} \frac{m_{\bar{B}_{s}}+m_{D_{s}^{*}}}{m_{\bar{B}_{s}}|\vec{q}|} V_{\lambda=-1}^{1}(|\vec{q}|) \\
& A_{+}\left(q^{2}\right)=i \frac{m_{\bar{B}_{s}}+m_{D_{s}^{*}}}{2 m_{\bar{B}_{s}}} \frac{m_{D_{s}^{*}}}{|\vec{q}| m_{\bar{B}_{s}}}\left\{-A_{\lambda=0}^{0}(|\vec{q}|)+\frac{m_{\bar{B}_{s}}-E_{D_{s}^{*}}(-\vec{q})}{|\vec{q}|} A_{\lambda=0}^{3}(|\vec{q}|)-\sqrt{2} \frac{m_{\bar{B}_{s}} E_{D_{s}^{*}}(-\vec{q})-m_{D_{s}^{*}}^{2}}{|\vec{q}| m_{D_{s}^{*}}} A_{\lambda=-1}^{1}(|\vec{q}|)\right\} \\
& A_{-}\left(q^{2}\right)=-i \frac{m_{\bar{B}_{s}}+m_{D_{s}^{*}}}{2 m_{\bar{B}_{s}}} \frac{m_{D_{s}^{*}}}{|\vec{q}| m_{\bar{B}_{s}}}\left\{A_{\lambda=0}^{0}(|\vec{q}|)+\frac{m_{\bar{B}_{s}}+E_{D_{s}^{*}}(-\vec{q})}{|\vec{q}|} A_{\lambda=0}^{3}(|\vec{q}|)-\sqrt{2} \frac{m_{\bar{B}_{s}} E_{D_{s}^{*}}(-\vec{q})+m_{D_{s}^{*}}^{2}}{|\vec{q}| m_{D_{s}^{*}}} A_{\lambda=-1}^{1}(|\vec{q}|)\right\} \\
& A_{0}\left(q^{2}\right)=-i \sqrt{2} \frac{1}{m_{\bar{B}_{s}}-m_{D_{s}^{*}}} A_{\lambda=-1}^{1}(|\vec{q}|)
\end{aligned}
$$



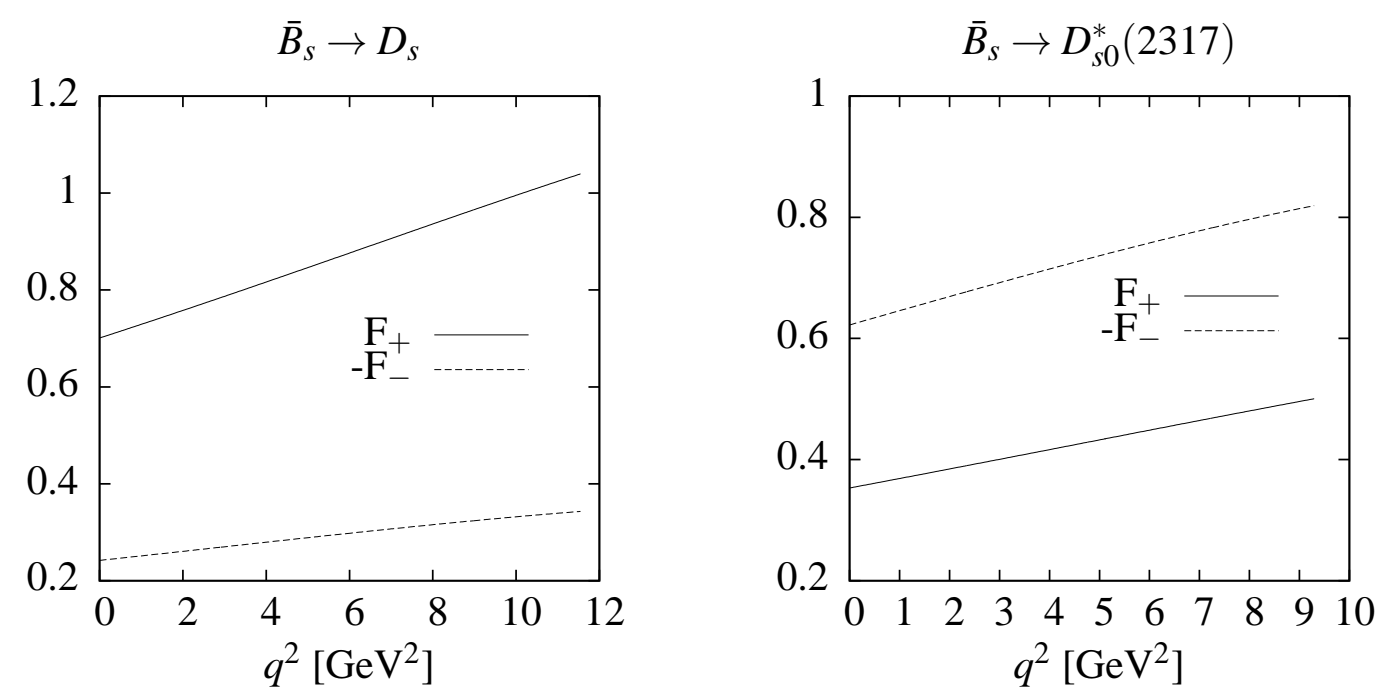

FIG. 1. Form factors for the semileptonic decay of $B_{s}$ mesons into $0^{-}$(left panel) or $0^{+}$(right panel) $c \bar{s}$ states.

with $V_{\lambda}^{\mu}(|\vec{q}|)$ and $A_{\lambda}^{\mu}(|\vec{q}|)$ calculated in our model as

$$
\begin{aligned}
& V_{\lambda}^{\mu}(|\vec{q}|)=\left\langle D_{s}^{*+}, \lambda-|\vec{q}| \vec{k}\left|J_{V}^{c b \mu}(0)\right| \bar{B}_{s}, \overrightarrow{0}\right\rangle=\sqrt{4 m_{\bar{B}_{s}} E_{D_{s}^{*}}(-\vec{q})}{ }_{\mathrm{NR}}\left\langle D_{s}^{*+}, \lambda-|\vec{q}| \vec{k}\left|J_{V}^{c b \mu}(0)\right| \bar{B}_{s}, \overrightarrow{0}\right\rangle_{\mathrm{NR}} \\
& A_{\lambda}^{\mu}(|\vec{q}|)=\left\langle D_{s}^{*+}, \lambda-|\vec{q}| \vec{k}\left|J_{A}^{c b \mu}(0)\right| \bar{B}_{s}, \overrightarrow{0}\right\rangle=\sqrt{4 m_{\bar{B}_{s}} E_{D_{s}^{*}}(-\vec{q})}{ }_{\mathrm{NR}}\left\langle D_{s}^{*+}, \lambda-|\vec{q}| \vec{k}\left|J_{A}^{c b \mu}(0)\right| \bar{B}_{s}, \overrightarrow{0}\right\rangle_{\mathrm{NR}}
\end{aligned}
$$

for which the remaining expressions can be found in the Appendix. The expressions for the axial vectors can be found from those in Eq. [15), by just replacing

$$
V_{\lambda}^{\mu}(|\vec{q}|) \leftrightarrow-A_{\lambda}^{\mu}(|\vec{q}|)
$$

Figures 2 and 3 shows the different form factors corresponding to semileptonic decays into vector and pseudovector states. These form factors have been calculated with the wave functions derived from the AL1 potential. In Fig 3 , the left (right) panel represents the form factors calculated for semileptonic decays into ${ }^{1} P_{1}\left({ }^{3} P_{1}\right)$ states.

\section{Decays into tensor and pseudotensor states}

For $\bar{B}_{s}$ mesons decaying into tensor states, the form factors can be evaluated as

$$
\begin{aligned}
& T_{1}\left(q^{2}\right)=-i \frac{2 m_{D_{s 2}^{2}}}{m_{\bar{B}_{s}}|\vec{q}|} A_{T \lambda=+1}^{1}(|\vec{q}|) \\
& T_{2}\left(q^{2}\right)=i \frac{1}{2 m_{\bar{B}_{s}}^{3}}\left\{-\sqrt{\frac{3}{2}} \frac{m_{D_{s 2}}^{2}}{|\vec{q}|^{2}} A_{T \lambda=0}^{0}(|\vec{q}|)-\sqrt{\frac{3}{2}} \frac{m_{D_{s 2}^{*}}^{2}}{|\vec{q}|^{3}}\left(E_{D_{s 2}^{*}}(-\vec{q})-m_{\bar{B}_{s}}\right) A_{T \lambda=0}^{3}(|\vec{q}|)+\frac{2 m_{D_{s 2}^{*}}}{|\vec{q}|}\left(1-\frac{E_{D_{s 2}^{*}}(-\vec{q})}{|\vec{q}|^{2}}\left(E_{D_{s 2}^{*}}(-\vec{q})-m_{\bar{B}_{s}}\right)\right)\right\} \\
& T_{3}\left(q^{2}\right)=i \frac{1}{2 m_{\bar{B}_{s}}^{3}}\left\{-\sqrt{\frac{3}{2}} \frac{m_{D_{s 2}}^{2}}{|\vec{q}|^{2}} A_{T \lambda=0}^{0}(|\vec{q}|)-\sqrt{\frac{3}{2}} \frac{m_{D_{s 2}}^{2}}{|\vec{q}|^{3}}\left(E_{D_{s 2}^{*}}(-\vec{q})+m_{\bar{B}_{s}}\right) A_{T \lambda=0}^{3}(|\vec{q}|)+\frac{2 m_{D_{s 2}}^{*}}{|\vec{q}|}\left(1-\frac{E_{D_{s 2}^{*}}(-\vec{q})}{|\vec{q}|^{2}}\left(E_{D_{s 2}^{*}}(-\vec{q})+m_{\bar{B}_{s}}\right)\right)\right\} \\
& T_{4}\left(q^{2}\right)=i \frac{m_{D_{s 2}}^{*}}{m_{\bar{B}_{s}}^{2}|\vec{q}|^{2}} A_{T \lambda=+1}^{1}(|\vec{q}|)
\end{aligned}
$$




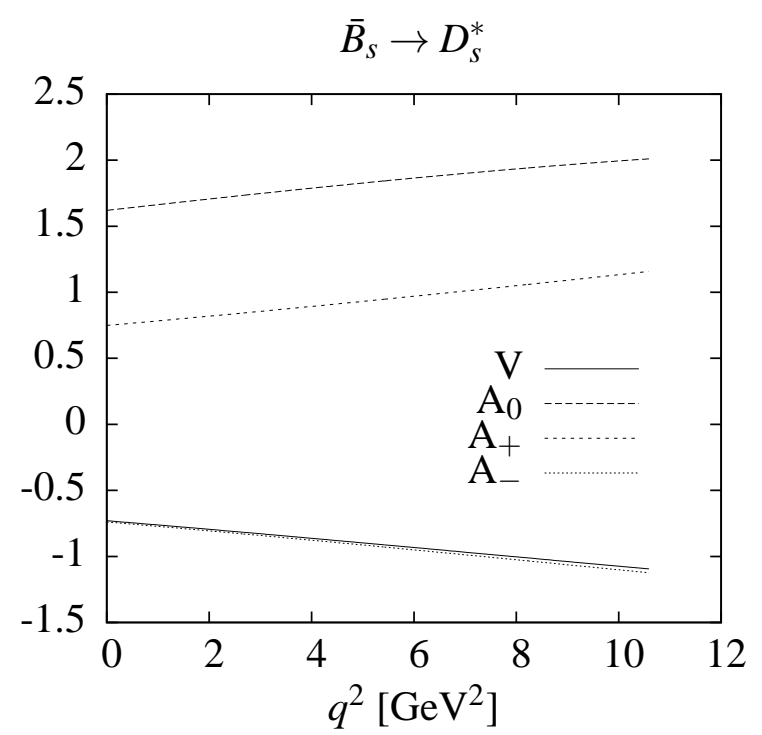

FIG. 2. Form factors for the decay of $B_{s}$ mesons into vector $D_{s}^{*}$ states.
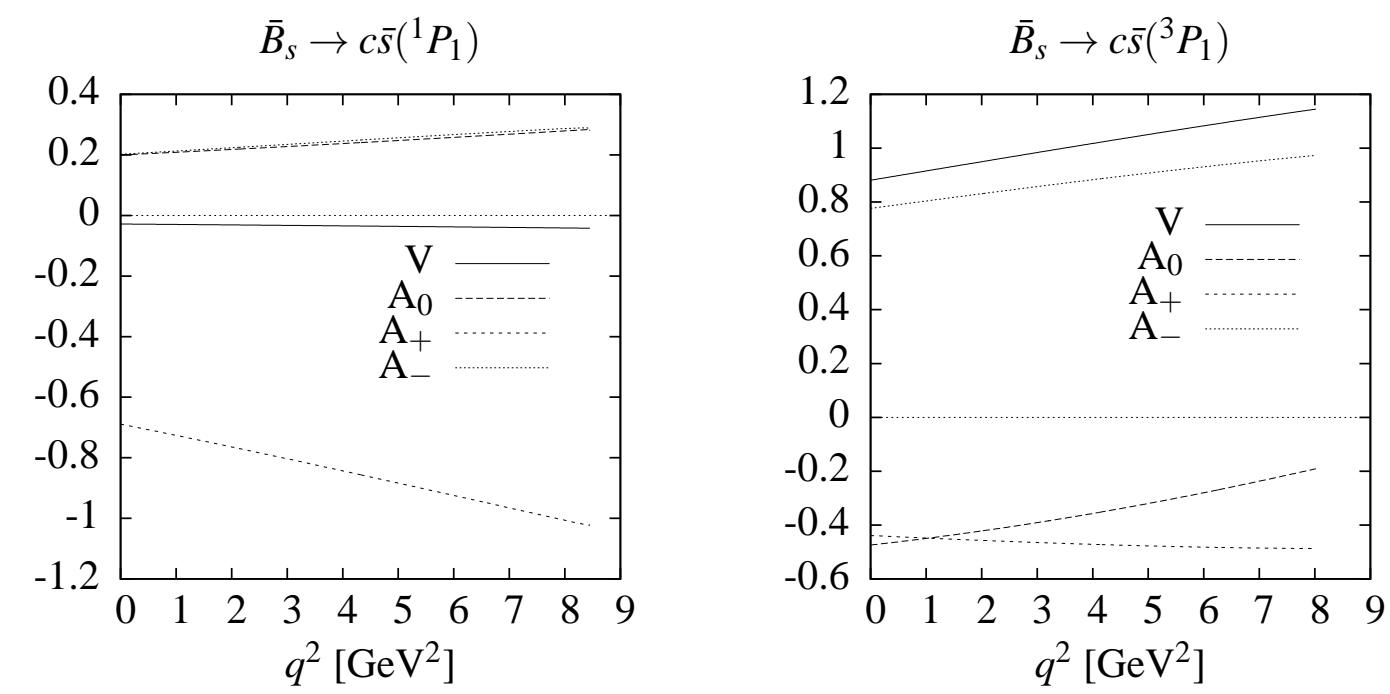

FIG. 3. Form factors for the decay of $B_{S}$ mesons into $c \bar{s}, J^{P}=1^{+}, S=0$ (left panel) and $c \bar{s}, J^{P}=1^{+}, S=1$ (right panel) states.

with $V_{T \lambda}^{\mu}(|\vec{q}|)$ and $A_{T \lambda}^{\mu}(|\vec{q}|)$ calculated in our model as

$$
\begin{aligned}
& V_{T \lambda}^{\mu}(|\vec{q}|)=\left\langle D_{s 2}^{*+}, \lambda-|\vec{q}| \vec{k}\left|J_{V}^{c b \mu}(0)\right| \bar{B}_{s}, \overrightarrow{0}\right\rangle=\sqrt{4 m_{\bar{B}_{s}} E_{D_{s 2}^{*}}(-\vec{q})}{ }_{\mathrm{NR}}\left\langle D_{s 2}^{*+}, \lambda-|\vec{q}| \vec{k}\left|J_{V}^{c b \mu}(0)\right| \bar{B}_{s}, \overrightarrow{0}\right\rangle_{\mathrm{NR}} \\
& A_{T \lambda}^{\mu}(|\vec{q}|)=\left\langle D_{s 2}^{*+}, \lambda-|\vec{q}| \vec{k}\left|J_{A}^{c b \mu}(0)\right| \bar{B}_{s}, \overrightarrow{0}\right\rangle=\sqrt{4 m_{\bar{B}_{s}} E_{D_{s 2}^{*}}(-\vec{q})}{ }_{\mathrm{NR}}\left\langle D_{s 2}^{*+}, \lambda-|\vec{q}| \vec{k}\left|J_{A}^{c b \mu}(0)\right| \bar{B}_{s}, \overrightarrow{0}\right\rangle_{\mathrm{NR}}
\end{aligned}
$$

for which the remaining expressions can be found in the Appendix. Again, the form factor corresponding to a decay into a pseudotensor state can be obtained from those above, just replacing

$$
V_{T \lambda}^{\mu}(|\vec{q}|) \leftrightarrow-A_{T \lambda}^{\mu}(|\vec{q}|)
$$


In Figure 4 we have represented the form factors corresponding to decays into tensor and pseudotensor states, with the wave functions of the AL1 potential.
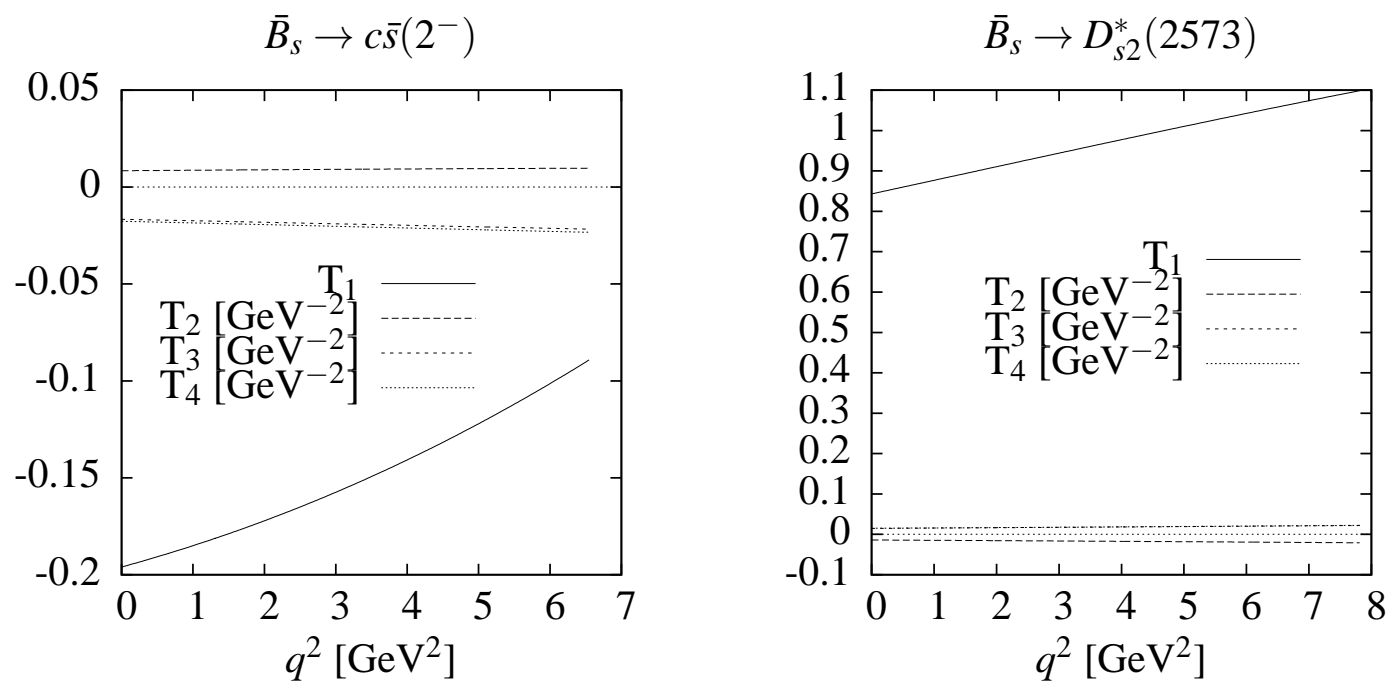

FIG. 4. Form factors for the decay of $B_{s}$ mesons into tensor (left panel) and pseudotensor (right panel) $c \bar{s}$ states.

\section{E. Decay width}

Let us consider the double differential decay width with respect to $q^{2}$ and the cosine, $x_{l}$, of the angle between the final meson momentum and the momentum of the final charged lepton, the latter measured in the lepton-neutrino center of mass frame (CMF). For a $\bar{B}_{s}$ at rest, this differential decay width results to be

$$
\frac{d^{2} \Gamma}{d x_{l} d q^{2}}=\frac{G_{F}^{2}}{64 m_{\bar{B}_{s}}^{2}} \frac{\left|V_{b c}\right|^{2}}{8 \pi^{3}} \frac{\lambda^{1 / 2}\left(q^{2}, m_{\bar{B}_{s}}^{2}, m_{c \bar{s}}^{2}\right)}{2 m_{\bar{B}_{s}}} \frac{q^{2}-m_{l}^{2}}{q^{2}} \mathcal{H}_{\alpha \beta}\left(P_{\bar{B}_{s}}, P_{c \bar{s}}\right) \mathcal{L}^{\alpha \beta}\left(p_{l}, p_{v}\right),
$$

where $G_{F}=1.16637(1) \times 10^{-5} \mathrm{GeV}^{-2}[39]$ is the Fermi constant, $\lambda(a, b, c)=(a+b-c)^{2}-4 a b, m_{l}$ is the mass of the charged lepton, $\mathcal{H}$ and $\mathcal{L}$ are the hadron and lepton tensors, and $P_{\bar{B}_{s}}, P_{c \bar{s}}, p_{l}$ and $p_{v}$ are the meson and lepton four momenta.

The lepton tensor is

$$
\mathcal{L}^{\alpha \beta}\left(p_{l}, p_{v}\right)=8\left(p_{l}^{\alpha} p_{v}^{\beta}+p_{l}^{\beta} p_{v}^{\alpha}-g^{\alpha \beta} p_{l} \cdot p_{v} \mp i \varepsilon^{\alpha \beta \sigma \rho} p_{l \sigma} p_{v \rho}\right)
$$

where, in the last term, the minus (plus) sign corresponds to a decay into $l^{-} \overline{\mathrm{v}}_{l}\left(l^{+} \mathrm{v}_{l}\right)$. The hadron tensor is given by

$$
\mathcal{H}_{\alpha \beta}=\sum_{\lambda} h_{(\lambda) \alpha}\left(P_{\bar{B}_{s}}, P_{c \bar{s}}\right) h_{(\lambda) \beta}^{(*)}\left(P_{\bar{B}_{s}}, P_{c \bar{s}}\right)
$$

where

$$
h_{(\lambda) \alpha}\left(P_{\bar{B}_{s}}, P_{c \bar{s}}\right)=\left\langle c \bar{s}, \lambda \vec{P}_{c \bar{s}}\left|J_{\alpha}^{c b}\right| \bar{B}_{s} \vec{P}_{\bar{B}_{s}}\right\rangle .
$$

is just the corresponding matrix element of the $b \rightarrow c$ V-A weak current given in Eq. 8

To evaluate the scalar

$$
\mathcal{H}_{\alpha \beta}\left(P_{\bar{B}_{s}}, P_{c \bar{s}}\right) \mathcal{L}^{\alpha \beta}\left(p_{l}, p_{v}\right)
$$


we choose $\vec{P}_{c \bar{s}}$ to be along the negative $z$-axis, which involves that the lepton CMF moves along the positive $z$-axis.

To proceed with the calculation we shall follow [38] and introduce the helicity components for the hadron and lepton tensor and rewrite the scalar of the expression of Eq. (25) as

$$
\mathcal{H}_{\alpha \beta}\left(P_{\bar{B}_{s}}, P_{c \bar{s}}\right) \mathcal{L}^{\alpha \beta}\left(p_{l}, p_{v}\right)=\mathcal{H}^{\sigma \rho}\left(P_{\bar{B}_{s}}, P_{c \bar{s}}\right) g_{\sigma \alpha} g_{\beta \rho} \mathcal{L}^{\alpha \beta}\left(p_{l}, p_{v}\right)
$$

where [43]

$$
\begin{aligned}
g_{\mu v} & =\sum_{r=t, \pm 1,0} g_{r r} \varepsilon_{(r) \mu}(q) g_{r r} \varepsilon_{(r) v}^{(*)}(q) \\
g_{t t} & =1, \quad g_{ \pm 1,0}=-1
\end{aligned}
$$

with $\varepsilon_{(t)}^{\mu}(q)=q^{\mu} / q^{2}$ and $\varepsilon_{(r)}(q), r= \pm 1,0$ are the polarization vector for an on-shell particle with four momentum $q$ and polarization $r$.

We shall define the helicity components of the hadron and lepton tensors as

$$
\begin{aligned}
\mathcal{H}_{r s}\left(P_{\bar{B}_{s}}, P_{c \bar{s}}\right) & =\varepsilon_{(r) \sigma}^{*}(q) \mathcal{H}^{\sigma \rho}\left(P_{\bar{B}_{s}}, P_{c \bar{s}}\right) \varepsilon_{(s) \rho}(q) \\
\mathcal{L}_{r s}\left(p_{l}, p_{v}\right) & =\varepsilon_{(r) \sigma}(q) \mathcal{L}^{\sigma \rho}\left(p_{l}, p_{v}\right) \varepsilon_{(s) \rho}^{*}(q) .
\end{aligned}
$$

The contraction of lepton and hadron tensors is, using the expressions above

$$
\mathcal{H}_{\alpha \beta}\left(P_{\bar{B}_{s}}, P_{c \bar{s}}\right) \mathcal{L}^{\alpha \beta}\left(p_{l}, p_{v}\right)=\sum_{r, s=t, \pm 1,0} g_{r r} g_{s s} \mathcal{H}_{r s}\left(P_{\bar{B}_{s}}, P_{c \bar{s}}\right) \mathcal{L}_{r s}\left(p_{l}, p_{v}\right)
$$

We take advantage of the fact that the Wigner rotation relating the original frame and the CMF of the final leptons is the identity. In the latter, we have

$$
\mathcal{L}_{r s}\left(p_{l}, p_{v}\right)=\varepsilon_{(r) \alpha}(q) \mathcal{L}^{\alpha \beta}\left(p_{l}, p_{v}\right) \varepsilon_{(s) \beta}(q)=\varepsilon_{(r) \alpha}(\tilde{q}) \mathcal{L}^{\alpha \beta}\left(\tilde{p}_{l}, \tilde{p}_{v}\right) \varepsilon_{(s) \beta}(\tilde{q})
$$

where the tilde stands for the momentum measured in the leptons CMF. For evaluation, we take 2

$$
\begin{aligned}
& \tilde{p}_{l}^{\alpha}=\left(E_{l}\left(\left|\tilde{p}_{l}\right|\right),\left|\tilde{p}_{l}\right| \sqrt{\left(1-x_{l}^{2}\right)}, 0,\left|\tilde{p}_{l}\right| x_{l}\right) \\
& \tilde{p}_{v}^{\alpha}=\left(\left|\tilde{p}_{l}\right|,\left|\tilde{p}_{l}\right| \sqrt{\left(1-x_{l}^{2}\right)}, 0,\left|\tilde{p}_{l}\right| x_{l}\right)
\end{aligned}
$$

where $\left|\tilde{p}_{l}\right|$ is the modulus of the lepton three momentum in the leptons CMF. Now let us evaluate the lepton tensor helicity components that we need.

$$
\begin{aligned}
\mathcal{L}_{t t}\left(p_{l}, p_{v}\right) & =4 \frac{m_{l}^{2}\left(q^{2}-m^{2}\right)}{q^{2}} \\
\mathcal{L}_{t 0}\left(p_{l}, p_{v}\right) & =-4 x_{l} \frac{m_{l}^{2}\left(q^{2}-m^{2}\right)}{q^{2}} \\
\mathcal{L}_{+1+1}\left(p_{l}, p_{v}\right) & =\left(q^{2}-m^{2}\right)\left(4\left(1 \pm x_{l}\right)-2\left(1-x_{l}^{2}\right) \frac{\left(q^{2}-m^{2}\right)}{q^{2}}\right) \\
\mathcal{L}_{-1-1}\left(p_{l}, p_{v}\right) & =\left(q^{2}-m^{2}\right)\left(4\left(1 \mp x_{l}\right)-2\left(1-x_{l}^{2}\right) \frac{\left(q^{2}-m^{2}\right)}{q^{2}}\right) \\
\mathcal{L}_{00}\left(p_{l}, p_{v}\right) & =4\left(q^{2}-m^{2}\right) \frac{1-x_{l}\left(q^{2}-m^{2}\right)}{q^{2}}
\end{aligned}
$$

As for the hadron tensor, we introduce the helicity amplitudes defined as

$$
h_{(\lambda) r}\left(P_{\bar{B}_{s}}, P_{c \bar{s}}\right)=\varepsilon_{(r) \alpha}^{*} h_{(\lambda)}^{\alpha}\left(P_{\bar{B}_{s}}, P_{c \bar{s}}\right),
$$

in terms of which the hadron tensor can be written as

$$
\mathcal{H}_{r s}\left(P_{\bar{B}_{s}}, P_{c \bar{s}}\right)=\sum_{\lambda} h_{(\lambda) r}\left(P_{\bar{B}_{s}}, P_{c \bar{s}}\right) h_{(\lambda) s}^{*}\left(P_{\bar{B}_{s}}, P_{c \bar{s}}\right)
$$

\footnotetext{
${ }^{2}$ As we have taken the momentum of the final meson in the negative $z$ direction, this is in accordance with the definition of $x_{l}$.
} 
The expressions for the helicity amplitudes in the original frame are given as [38, 40]:

- Transitions to scalar states

$$
\begin{aligned}
h_{t}\left(P_{\bar{B}_{s}}, P_{c \bar{s}}\right) & =\frac{m_{\bar{B}_{s}}^{2}-m_{c \bar{s}}^{2}}{\sqrt{q^{2}}} F_{+}\left(q^{2}\right)+\sqrt{q^{2}} F_{-}\left(q^{2}\right) \\
h_{0}\left(P_{\bar{B}_{s}}, P_{c \bar{s}}\right) & =\frac{\lambda^{1 / 2}\left(q^{2}, m_{\bar{B}_{s}}^{2}, m_{c \bar{s}}^{2}\right)}{\sqrt{q^{2}}} F_{+}\left(q^{2}\right) \\
h_{+1}\left(P_{\bar{B}_{s}}, P_{c \bar{s}}\right) & =h_{-1}\left(P_{\bar{B}_{s}}, P_{c \bar{s}}\right)=0
\end{aligned}
$$

- Transitions to vector states

$$
\begin{aligned}
h_{(\lambda) t}\left(P_{\bar{B}_{s}}, P_{c \bar{s}}\right) & =i \delta_{\lambda 0} \frac{\lambda^{1 / 2}\left(q^{2}, m_{\bar{B}_{s}}^{2}, m_{c \bar{s}}^{2}\right)}{2 m_{c \bar{s}} \sqrt{q^{2}}}\left(\left(m_{\bar{B}_{s}}-m_{c \bar{s}}\right)\left(A_{0}\left(q^{2}\right)-A_{+}\left(q^{2}\right)\right)-\frac{q^{2}}{m_{\bar{B}_{s}}+m_{c \bar{s}}} A_{-}\left(q^{2}\right)\right) \\
h_{(\lambda)+1}\left(P_{\bar{B}_{s}}, P_{c \bar{s}}\right) & =-i \delta_{\lambda-1}\left(\frac{\lambda^{1 / 2}\left(q^{2}, m_{\bar{B}_{s}}^{2}\right)}{m_{\bar{B}_{s}}+m_{c \bar{s}}} V\left(q^{2}\right)+\left(m_{\bar{B}_{s}}-m_{c \bar{s}}\right) A_{0}\left(q^{2}\right)\right) \\
h_{(\lambda)-1}\left(P_{\bar{B}_{s}}, P_{c \bar{s}}\right) & =-i \delta_{\lambda+1}\left(\frac{\lambda^{1 / 2}\left(q^{2}, m_{\bar{B}_{s}}^{2}\right)}{m_{\bar{B}_{s}}+m_{c \bar{s}}} V\left(q^{2}\right)+\left(m_{\bar{B}_{s}}-m_{c \bar{s}}\right) A_{0}\left(q^{2}\right)\right) \\
h_{(\lambda) 0}\left(P_{\bar{B}_{s}}, P_{c \bar{s}}\right) & =i \delta_{\lambda 0}\left(\left(m_{\bar{B}_{s}}-m_{c \bar{s}}\right) \frac{m_{\bar{B}_{s}}^{2}-q^{2}-m_{c \bar{s}}^{2}}{2 m_{c \bar{s}} \sqrt{q^{2}}} A_{0}\left(q^{2}\right)-\frac{\lambda\left(q^{2}, m_{\bar{B}_{s}}^{2}, m_{c \bar{s}}^{2}\right)}{2 m_{c \bar{s}} \sqrt{q^{2}}} \frac{A_{+}\left(q^{2}\right)}{m_{\bar{B}_{s}}+m_{c \bar{s}}}\right)
\end{aligned}
$$

- Transitions to tensor states

$$
\begin{aligned}
h_{(\lambda) t}\left(P_{\bar{B}_{s}}, P_{c \bar{s}}\right) & =-i \delta_{\lambda 0} \sqrt{\frac{2}{3}} \frac{\lambda\left(q^{2}, m_{\bar{B}_{s}}^{2}, m_{c \bar{s}}^{2}\right)}{4 m_{c \bar{s}}^{2} \sqrt{q^{2}}}\left(T_{1}\left(q^{2}\right)+\left(m_{\bar{B}_{s}}^{2}-m_{c \bar{s}}^{2}\right) T_{2}\left(q^{2}\right)+q^{2} T_{3}\left(q^{2}\right)\right) \\
h_{(\lambda)+1}\left(P_{\bar{B}_{s}}, P_{c \bar{s}}\right) & =i \delta_{\lambda-1} \frac{1}{\sqrt{2}} \frac{\lambda^{1 / 2}\left(q^{2}, m_{\bar{B}_{s}}^{2}, m_{c \bar{s}}^{2}\right)}{2 m_{c \bar{s}}}\left(T_{1}\left(q^{2}\right)-\lambda^{1 / 2}\left(q^{2}, m_{\bar{B}_{s}}^{2} m_{c \bar{s}}^{2}\right) T_{4}\left(q^{2}\right)\right) \\
h_{(\lambda)-1}\left(P_{\bar{B}_{s}}, P_{c \bar{s}}\right) & =i \delta_{\lambda+1} \frac{1}{\sqrt{2}} \frac{\lambda^{1 / 2}\left(q^{2}, m_{\bar{B}_{s}}^{2}, m_{c \bar{s}}^{2}\right)}{2 m_{c \bar{s}}}\left(T_{1}\left(q^{2}\right)+\lambda^{1 / 2}\left(q^{2}, m_{\bar{B}_{s}}^{2} m_{c \bar{s}}^{2}\right) T_{4}\left(q^{2}\right)\right) \\
h_{(\lambda) 0}\left(P_{\bar{B}_{s}}, P_{c \bar{s}}\right) & =-i \delta_{\lambda 0} \sqrt{\frac{2}{3}} \frac{\lambda^{1 / 2}\left(q^{2}, m_{\bar{B}_{s}}^{2}, m_{c \bar{s}}^{2}\right)}{4 m_{c \bar{s}}^{2} \sqrt{q^{2}}}\left(\left(m_{\bar{B}_{s}}^{2}-q^{2}-m_{c \bar{s}}\right) T_{1}\left(q^{2}\right)+\lambda\left(q^{2}, m_{\bar{B}_{s}}^{2}, m_{c \bar{s}}^{2}\right) T_{2}\left(q^{2}\right)\right)
\end{aligned}
$$

Where we shall remark that the helicity amplitudes, and thus the components of the hadron tensor depend only on $q^{2}$. We define the following combinations for further convenience:

$$
\begin{aligned}
H_{U} & =\mathcal{H}_{+1+1}+\mathcal{H}_{1-1} \\
H_{P} & =\mathcal{H}_{+1+1}-\mathcal{H}_{1-1} \\
H_{L} & =\mathcal{H}_{00} ; H_{S}=3 \mathcal{H}_{t t} ; H_{S L}=\mathcal{H}_{t 0} \\
\tilde{H}_{J} & =\frac{m_{l}^{2}}{2 q^{2}} \mathcal{H} ; \quad J=U, L, S, S L
\end{aligned}
$$

with $U, L, P, S$ and $S L$ representing, respectively, unpolarized-transverse, longitudinal, parity-odd, scalar and scalar-longitudinal interference.

The double differential decay width can be written in terms of the combination above as

$$
\begin{aligned}
\frac{d^{2} \Gamma}{d q^{2} d x_{l}} & =\frac{G_{F}^{2}}{8 \pi^{3}}\left|V_{b c}\right|^{2} \frac{\left(q^{2}-m_{l}^{2}\right)^{2}}{12 m_{\bar{B}_{s}}^{2} q^{2}} \frac{\lambda^{1 / 2}\left(q^{2}, m_{\bar{B}_{s}}^{2}, m_{c \bar{s}}^{2}\right)}{2 m_{\bar{B}_{s}}} \\
& \times\left\{\frac{3}{8}\left(1+x_{l}^{2}\right) H_{U}+\frac{3}{4}\left(1-x_{l}^{2}\right) H_{L} \pm \frac{3}{4} H_{P}+\frac{3}{4}\left(1-x_{l}^{2}\right) \tilde{H}_{U}+\frac{3}{2} x_{l}^{2} \tilde{H}_{l}+\frac{1}{2} \tilde{H}_{S}+3 x_{l} \tilde{H}_{s l}\right\}
\end{aligned}
$$


The term $H_{P}$ changes sign for antiparticle decay, in contrast to the rest of the helicity components. This extra sign compensates the $\mp$ sign in the lepton tensor, leading to an expression for the double differential decay which is the same for particle or antiparticle decay.

Finally, we obtain the differential decay width integrating over $x_{l}$.

$$
\frac{d \Gamma}{d q^{2}}=\frac{G_{F}^{2}}{8 \pi^{3}}\left|V_{b c}\right|^{2} \frac{\left(q^{2}-m_{l}^{2}\right)^{2}}{12 m_{\bar{B}_{s}}^{2} q^{2}} \frac{\lambda^{1 / 2}\left(q^{2}, m_{\bar{B}_{s}}^{2}, m_{c \bar{s}}^{2}\right)}{2 m_{\bar{B}_{s}}}\left\{H_{U}+H_{L}+\tilde{H}_{U}+\tilde{H}_{L}+\tilde{H}_{S}\right\},
$$

from where we obtain the total decay width integrating over $q^{2}$, that can be written as

$$
\Gamma=\Gamma_{U}+\Gamma_{L}+\tilde{\Gamma}_{U}+\tilde{\Gamma}_{L}+\tilde{\Gamma}_{S}
$$

with $\Gamma$ and $\Gamma_{J}$ partial helicity widths defined as

$$
\Gamma_{J}=\int d q^{2} \frac{G_{F}^{2}}{8 \pi^{3}}\left|V_{b c}\right|^{2} \frac{\left(q^{2}-m_{l}^{2}\right)^{2}}{12 m_{\bar{B}_{s}}^{2} q^{2}} \frac{\lambda^{1 / 2}\left(q^{2}, m_{\bar{B}_{s}}^{2}, m_{c \bar{s}}^{2}\right)}{2 m_{\bar{B}_{s}}} H_{J}
$$

and similarly for $\tilde{\Gamma}_{J}$ in terms of $\tilde{H}_{J}$.

The forward-backward asymmetry of the charged leptons, measured in the leptons CMF, which in terms of partial helicity widths, can be written as

$$
A_{F B}=\frac{\Gamma_{x_{l}>0}-\Gamma_{x_{l}<0}}{\Gamma_{x_{l}>0}+\Gamma_{x_{l}<0}}=\frac{3}{4} \frac{ \pm \Gamma_{P}+4 \tilde{\Gamma}_{S L}}{\Gamma_{U}+\Gamma_{L}+\tilde{\Gamma}_{U}+\tilde{\Gamma}_{L}+\tilde{\Gamma}_{S}}
$$

As $\Gamma_{P}$ changes sign for antiparticle decay, $A_{F B}$ is the same for a negative charged lepton as for a positive.

\section{F. Results}

Table III summarizes the values for the total decay widths calculated with our model. We give the semileptonic decay widths for the different leptons in the final state, in units of $10^{-15} \mathrm{GeV}$. The central values have been calculated using the AL1 potential of [42], while the theoretical uncertainties have been estimated by considering other potential models (see Ref. [42]). Table IV] shows the corresponding values for branching fractions.

In Table V] we compare with previous results. In Refs. [28] the authors adopt the relativistic quark model. Chen et al. solve the instantaneous Bethe-Salpeter equation in [32] to estimate the weak transition form factors. In [33] the authors work out the form factors within the covariant light front quark model. Azizi et al. in [34, 35] and Blasi et al. in [36] apply the sum rules technique to obtain the form factors and branching fractions. In [37], work within the Constituent Quark Model, as in this work. In Ref. [44] we studied some of the decays into orbitally excited final $D_{s}$ states, using the potential model of [45].

The results of this work are in a systematic good agreement with those from the relativistic quark model of [28]. The agreement is also good with the quark model calculation of [37]. It is worth to mention that our results for decays into orbitally excited final $D_{s}$ mesons are in rather good agreement with our previous results from [44], though in that work the potential model that have been used is much more sophisticated, even enabling the posibility to consider non- $q \bar{q}$ components for these orbitally excited states. Our results also compare well to the sum-rules calculation of [34, 35], while the result of [36] is lower by about one half. The same happens if we compare with the results of [32] or [33].

In Tables VI and VII we give our results for partial helicity widths corresponding to $\bar{B}_{s}^{0}$, and the values we obtain for the forward-backward asymetry, respectively. In Table VI the "P" column changes sign for $B_{s}^{0}$ decay. As before, the central values have been evaluated with the AL1 potential.

In the different panels of Figures 5 to 8 we plot the differential decay widths that we obtain for the different $J^{P} c \bar{s}$ final states, with $e^{+}$or $\tau^{+}$, accounting for the leptons.

\section{G. Heavy Quark Symmetry}

In systems with a quark with mass much larger than the QCD scale $\left(\Lambda_{\mathrm{QCD}}\right)$, the dynamics of the light degrees of freedom becomes independent of the heavy quark flavor and spin. 


\begin{tabular}{c|ccc}
\hline \hline $\bar{B}_{S} \rightarrow M^{\prime} l^{-} \overline{\mathrm{v}}_{l}$ & \multicolumn{3}{|c}{$\Gamma\left[10^{-15} \mathrm{GeV}\right]$} \\
\hline$M^{\prime}$ & $l=e$ & $l=\mu$ & $l=\tau$ \\
\hline$D_{s}^{+}$ & $10.37_{-0.2}^{+0.15}$ & $10.32_{-0.10}^{+0.16}$ & $2.99_{-0.03}^{+0.01}$ \\
$D_{s 0}^{*+}$ & $1.75_{-0.07}^{+0.03}$ & $1.74_{-0.08}^{+0.03}$ & $0.20_{-0.003}^{+0.003}$ \\
$D_{s}^{*+}$ & $28.02_{-0.48}^{+0.24}$ & $27.90_{-0.48}^{+0.86}$ & $6.86_{-0.09}^{+0.12}$ \\
$D_{s 1}^{+}(2460)$ & $2.07_{-0.09}$ & $2.05_{-0.08}$ & $0.17_{-0.008}$ \\
$D_{s 1}^{+}(2536)$ & $1.40_{-0.07}$ & $1.39_{-0.07}$ & $0.12_{-0.006}$ \\
$c \bar{s}\left(2^{-}\right)$ & $4.11_{-0.56} 10^{-2}$ & $4.06_{-0.64} 10^{-2}$ & $9.02_{-2.39} 10^{-4}$ \\
$D_{s 2}^{*+}$ & $1.97_{-0.15}$ & $1.95_{-0.14}$ & $0.12_{-0.02}$ \\
\hline
\end{tabular}

TABLE III. Decay widths in units of $10^{-15} \mathrm{GeV}$ for semileptonic $\bar{B}_{S} \rightarrow c \bar{s}$ decays. The central value has been obtained with the AL1 potential.

\begin{tabular}{c|cc}
\hline \hline$M^{\prime}$ & $l=e, \mu$ & $l=\tau$ \\
\hline$D_{s}^{+}$ & 2.32 & 0.67 \\
$D_{s 0}^{*+}$ & 0.39 & 0.04 \\
$D_{s}^{*+}$ & 6.26 & 1.53 \\
$D_{s 1}^{+}(2460)$ & 0.47 & 0.04 \\
$D_{s 1}^{+}(2536)$ & 0.32 & 0.03 \\
$c \bar{s}\left(2^{-}\right)$ & $9.210^{-3}$ & $2.010^{-4}$ \\
$D_{s 2}^{*+}$ & 0.44 & 0.03 \\
\hline
\end{tabular}

TABLE IV. Branching fractions for the indicated decay channels, in percentage.

The six form factors involved in the $\bar{B}_{S}$ decays into pseudoscalar and vector mesons are related by HQS, which reduces their evaluation to that of a single function, $\xi$. In particular, HQS predicts [20, 21]:

$$
\begin{aligned}
& h_{+}(\omega)=h_{V}(\omega)=h_{A_{1}}(\omega)=h_{A_{3}}(\omega)=\xi(\omega) \\
& h_{-}(\omega)=h_{A_{2}}(\omega)=0 .
\end{aligned}
$$

The $h$ form factors are just a redefinition of the those above, given by

$$
h_{ \pm}(\omega)=\frac{2 m_{\bar{B}_{s}}}{\sqrt{2 m_{\bar{B}_{s}} m_{c \bar{s}}}} f_{ \pm}(\omega)
$$

\begin{tabular}{|c|c|c|c|c|c|c|c|c|}
\hline & This work & [28] & [32] & [33] & [36] & [37] & {$[34],[35]$} & [44] \\
\hline $\bar{B}_{s} \rightarrow D_{s}^{+} e^{-} \overline{\mathrm{v}}_{e}$ & 2.32 & $2.1 \pm 0.2$ & $1.4-1.7$ & $1.0_{-0.3}^{+0.4}$ & $1.35 \pm 0.21$ & $2.73-3.00$ & $2.8-3.8$ & \\
\hline $\bar{B}_{s} \rightarrow D_{s}^{*+} e^{-} \overline{\mathrm{v}}_{e}$ & 6.26 & $5.3 \pm 0.5$ & $5.1-5.8$ & & $2.5 \pm 0.1$ & $7.49-7.66$ & $1.89-6.61$ & \\
\hline $\bar{B}_{S} \rightarrow D_{S}^{+} \tau^{-} \bar{v}_{\tau}$ & 0.67 & $0.62 \pm 0.05$ & $0.47-0.55$ & $0.33_{-0.11}^{+0.14}$ & & & & \\
\hline $\bar{B}_{s} \rightarrow D_{s}^{*+} \tau^{-} \bar{v}_{\tau}$ & 1.53 & $1.3 \pm 0.1$ & $1.2-1.3$ & & & & & \\
\hline $\bar{B}_{S} \rightarrow D_{s 0}^{*+} \mu^{-} \bar{v}_{\mu}$ & 0.39 & & & & & & & 0.44 \\
\hline $\bar{B}_{s} \rightarrow D_{s 1}^{*+}(2460) \mu^{-} \bar{v}_{\mu}$ & 0.47 & & & & & & & $0.17-0.5$ \\
\hline $\bar{B}_{s} \rightarrow D_{s 1}^{*+}(2536) \mu^{-} \bar{v}_{\mu}$ & 0.32 & & & & & & & 0.4 \\
\hline $\bar{B}_{s} \rightarrow D_{s 2}^{*+} \mu^{-} \bar{v}_{\mu}$ & 0.44 & & & & & & & 0.37 \\
\hline
\end{tabular}

TABLE V. Branching fractions for the indicated decay channels, in percentage. 


\begin{tabular}{|c|c|c|c|c|c|c|c|}
\hline & $1_{U}$ & $\tilde{\Gamma}_{U}$ & $\Gamma_{L}$ & $\tilde{\Gamma}_{L}$ & $\Gamma_{P}$ & $\tilde{\Gamma}_{S}$ & $\Gamma_{S L}$ \\
\hline s. & 0 & 0 & & $2.2910^{-6}$ & 0 & $4310^{-6}$ & 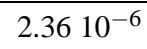 \\
\hline$e^{-} \bar{v}_{e}$ & 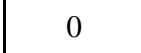 & 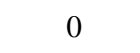 & 75 & 821 & 0 & $4410^{-6}$ & -7 \\
\hline $\bar{B}_{s} \rightarrow D_{s}^{*+} e^{-} \bar{v}_{e}$ & 13.87 & $4.1510^{-7}$ & 14.16 & $2.1310^{-6}$ & -7.32 & $9010^{-6}$ & $2.0310^{-6}$ \\
\hline 60)e & 32 & $1.610^{-8}$ & 75 & 411 & -0.22 & $9810^{-6}$ & $6.5110^{-7}$ \\
\hline$s-$ & 0.56 & $2.9710^{-8}$ & 0.84 & $3.0410^{-7}$ & -0.44 & $4010^{-7}$ & $0810^{-7}$ \\
\hline${ }_{s} \rightarrow c \bar{s}\left(2^{-}\right) e^{-} \bar{v}_{e}$ & $3.9510^{-2}$ & $3.3710^{-9}$ & $1.5810^{-3}$ & $3.5510^{-10}$ & $-3.2410^{-2}$ & $8.7610^{-10}$ & $3.1510^{-10}$ \\
\hline${ }_{2}^{+} e^{-} \bar{v}_{e}$ & 0.67 & $3.7610^{-8}$ & 1.30 & $4.3510^{-7}$ & -0.35 & $1.2510^{-6}$ & $4.2410^{-7}$ \\
\hline & & 0 & 10.11 & 4.7 & & ). & \\
\hline$-\bar{v}_{\mu}$ & 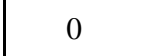 & 0 & 70 & .471 & & 010 & \\
\hline$-\bar{v}_{\mu}$ & 13.80 & .7410 & 13.91 & 4.681 & -7. & 0.12 & \\
\hline $\bar{\beta}_{s} \rightarrow D_{s 1}^{+}$ & 0 & $6.610^{-4}$ & 68 & 1.71 & -0 & -2 & 1.22 \\
\hline$-\bar{v}_{\mu}$ & 0.55 & $1.2310^{-3}$ & 0.81 & $5.5810^{-3}$ & -0. & $7910^{-3}$ & $5.7610^{-3}$ \\
\hline$\mu^{-} \bar{v}_{\mu}$ & $3.8910^{-2}$ & $1.3710^{-4}$ & $1.5510^{-3}$ & $7.4910^{-6}$ & $-3.2010^{-2}$ & $1.4610^{-5}$ & $5.8210^{-6}$ \\
\hline$\underline{\bar{B}_{s} \rightarrow D_{s 2}^{*+} \mu^{-} \bar{v}_{\mu}}$ & 0.66 & $1.5510^{-3}$ & 1.26 & $8.1510^{-3}$ & -0.35 & $2010^{-2}$ & $7.6910^{-3}$ \\
\hline & & & 0.9 & 0.2 & & & 0. \\
\hline$-\bar{v}_{\tau}$ & ( & 0 & $.2810^{-2}$ & 2.51 & & $2610^{-2}$ & $2.6110^{-2}$ \\
\hline $\bar{B}_{s} \rightarrow D_{s}^{*+} \tau^{-} \bar{v}_{\tau}$ & 3.18 & 0.68 & 2.06 & 0.46 & -1.3 & 0.49 & 0.26 \\
\hline $\bar{B}_{s}$ & 0.03 & $8.2210^{-3}$ & $5.1910^{-2}$ & $1.5010^{-2}$ & -1.71 & 0.0 & $1.8810^{-2}$ \\
\hline $\bar{B}_{s} \rightarrow D_{s 1}^{+}(2536) \tau^{-} \bar{v}_{\tau}$ & $4.4810^{-2}$ & $1.2510^{-2}$ & $2.6010^{-2}$ & $7.4910^{-3}$ & $-3.3910^{-2}$ & $3.5310^{-2}$ & $8.9610^{-3}$ \\
\hline $\bar{B}_{s} \rightarrow c \bar{s}\left(2^{-}\right) \tau^{-} \bar{v}_{\tau}$ & $6.2610^{-4}$ & $2.1410^{-4}$ & $4.4210^{-5}$ & $1.4410^{-5}$ & $-5.3410^{-4}$ & $3.0510^{-8}$ & $3.6810^{-6}$ \\
\hline $\bar{B}_{s} \rightarrow D_{s 2}^{*}$ & $4.2610^{-2}$ & $1.2510^{-2}$ & $3.8510^{-2}$ & $1.1510^{-2}$ & $-1.7510^{-2}$ & $1.2210^{-2}$ & $6.7310^{-3}$ \\
\hline
\end{tabular}

TABLE VI. Partial helicity widths in units of $10^{-15} \mathrm{GeV}$. These results have been calculated using the AL1 potential.

\begin{tabular}{l|ccc}
\hline \hline & \multicolumn{3}{|c}{$A_{\mathrm{FB}}$} \\
& $l=e$ & $l=\mu$ & $l=\tau$ \\
\hline $\bar{B}_{s} \rightarrow D_{s}^{+} l^{-} \overline{\mathrm{v}}_{l}$ & $6.8610^{-7}$ & $1.4710^{-2}$ & 0.36 \\
$\bar{B}_{s} \rightarrow D_{s 0}^{*+} l^{-} \overline{\mathrm{v}}_{l}$ & $8.2210^{-7}$ & $1.6210^{-2}$ & 0.39 \\
$\bar{B}_{s} \rightarrow D_{s}^{*+} l^{-} \overline{\mathrm{v}}_{l}$ & -0.20 & -0.19 & $-3.7110^{-2}$ \\
$\bar{B}_{s} \rightarrow D_{s 1}^{+}(2460) l^{-} \overline{\mathrm{v}}_{l}$ & -0.19 & -0.18 & 0.10 \\
$\bar{B}_{s} \rightarrow D_{s 1}^{+}(2536) l^{-} \overline{\mathrm{v}}_{l}$ & -0.41 & -0.40 & -0.20 \\
$\bar{B}_{s} \rightarrow c \bar{s}\left(2^{-}\right) l^{-} \overline{\mathrm{v}}_{l}$ & -0.59 & -0.59 & -0.43 \\
$\bar{B}_{s} \rightarrow D_{s 2}^{*+} l^{-} \overline{\mathrm{v}}_{l}$ & -0.14 & -0.12 & $6.0310^{-2}$ \\
\hline
\end{tabular}

TABLE VII. Forward-Backward asymmetry parameters for the semileptonic $B_{s}$ decays, obtained for the AL1 potential.

for decays into pseudoscalar states, and

$$
\begin{aligned}
h_{V}(\omega) & =\sqrt{2} \sqrt{\frac{M_{D_{s}^{*}}}{M_{\bar{B}_{s}}}} \frac{V_{\lambda=-1}^{2}(|\vec{q}|)}{|\vec{q}|} \\
h_{A_{1}}(\omega) & =i \frac{\sqrt{2}}{w+1} \frac{1}{\sqrt{M_{\bar{B}_{s}} M_{D_{s}^{*}}}} A_{\lambda=-1}^{1}(|\vec{q}|) \\
h_{A_{2}}(\omega) & =i \sqrt{\frac{M_{D_{s}^{*}}}{M_{\bar{B}_{s}}}}\left(-\frac{A_{\lambda=0}^{0}(|\vec{q}|)}{|\vec{q}|}+\frac{E_{D_{s}^{*}}(|\vec{q}|) A_{\lambda=0}^{3}(|\vec{q}|)}{|\vec{q}|^{2}}-\sqrt{2} M_{D^{*}} \frac{A_{\lambda=-1}^{1}(|\vec{q}|)}{|\vec{q}|^{2}}\right) \\
h_{A_{3}}(\omega) & =i \frac{M_{D_{s}^{*}}^{2}}{\sqrt{M_{D_{s}^{*}} M_{\bar{B}_{s}}}}\left(-\frac{A_{\lambda=0}^{3}(|\vec{q}|)}{|\vec{q}|^{2}}+\frac{\sqrt{2}}{M_{D^{*}}} \frac{A_{\lambda=-1}^{1}(|\vec{q}|)}{|\vec{q}|^{2}}\right)
\end{aligned}
$$

for decays into vector states [23]. 

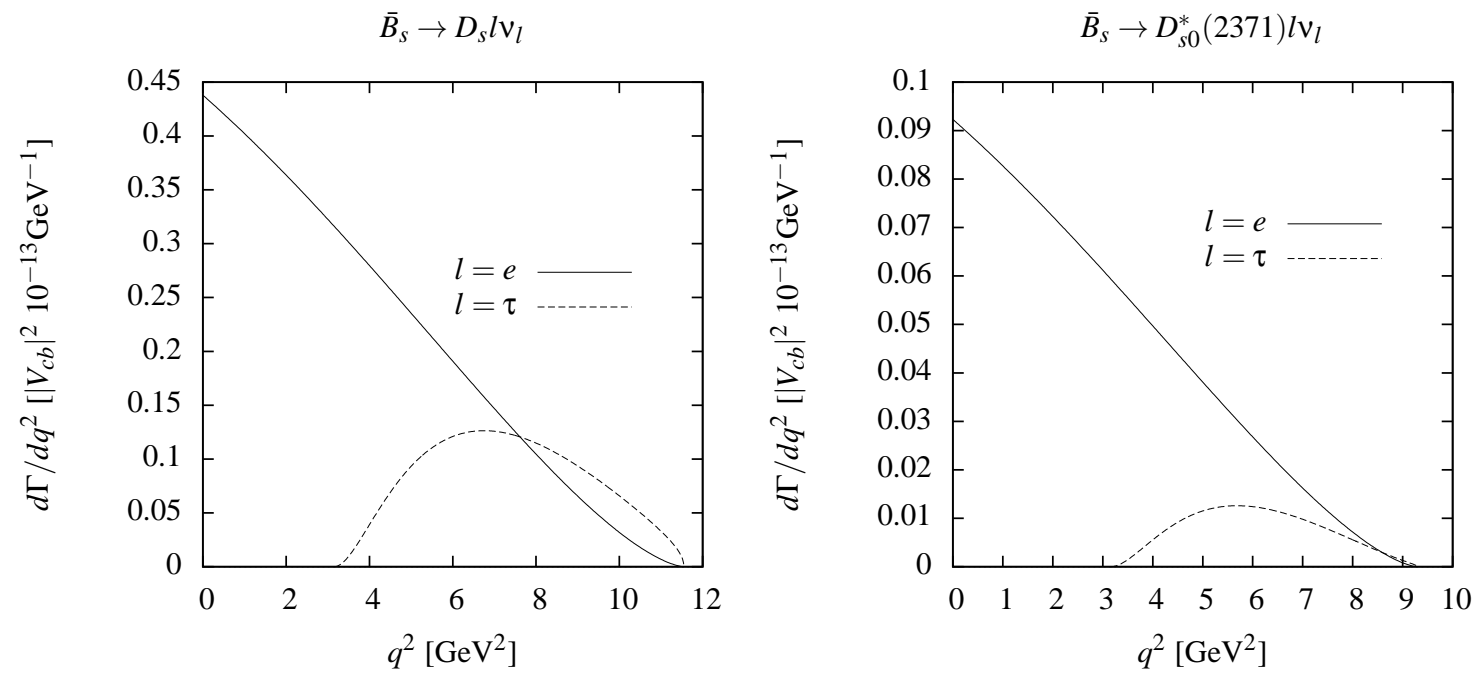

FIG. 5. Differential decay width for the $\bar{B}_{s}$ into $0^{-}$(left panel) and $0^{+}$(right panel) states.

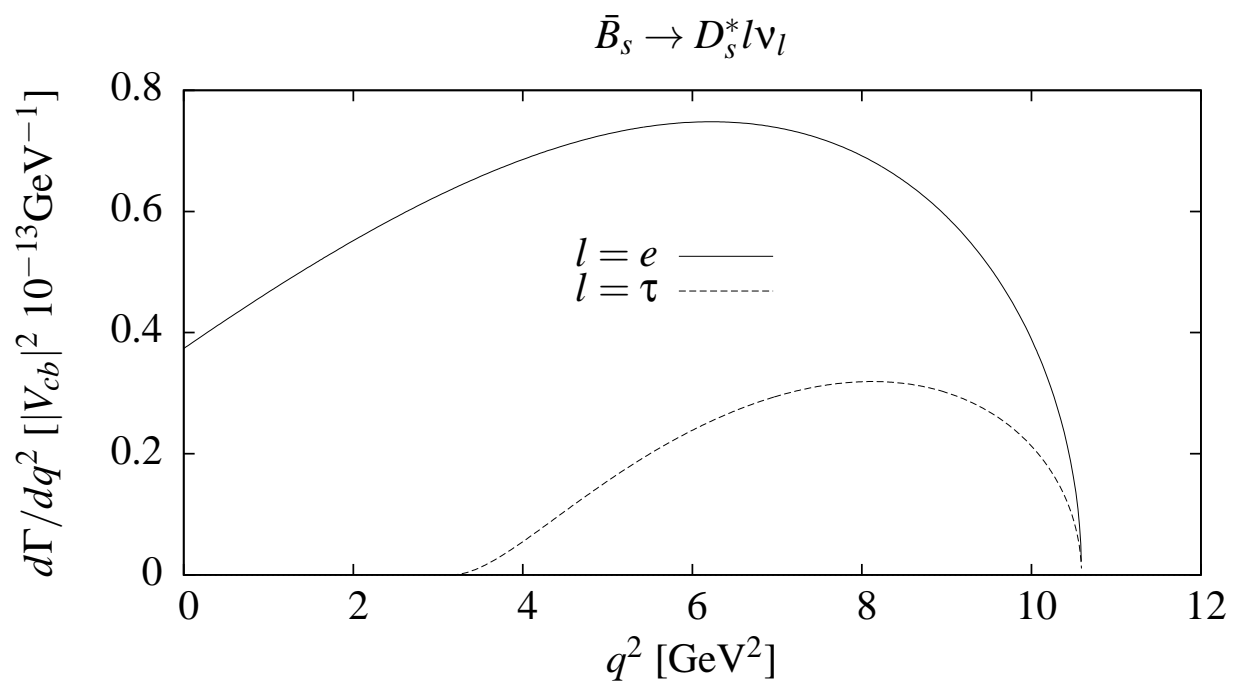

FIG. 6. Differential decay width for the semileptonic $\bar{B}_{s} \rightarrow D^{*}$ process.

Conservation of vector current in the equal mass case, provides another constrain, in the form of a normalization condition:

$$
\xi(\omega=1)=1 .
$$

The purpose of this section is test the form factors we have obtained previously against the HQS predictions. In the left panel of Fig. 9 we plot our values for the $h$ form factors. These values have been obtained with the wave functions of the AL1 potential. In the right panel of Fig. 9 , we also evaluate the ratios

$$
\begin{aligned}
& R_{1}(\omega)=\frac{h_{V}(\omega)}{h_{A_{1}}(\omega)} \\
& R_{2}(\omega)=\frac{h_{A_{3}}(\omega)+r h_{A_{2}}(\omega)}{h_{A_{1}}(\omega)}
\end{aligned}
$$



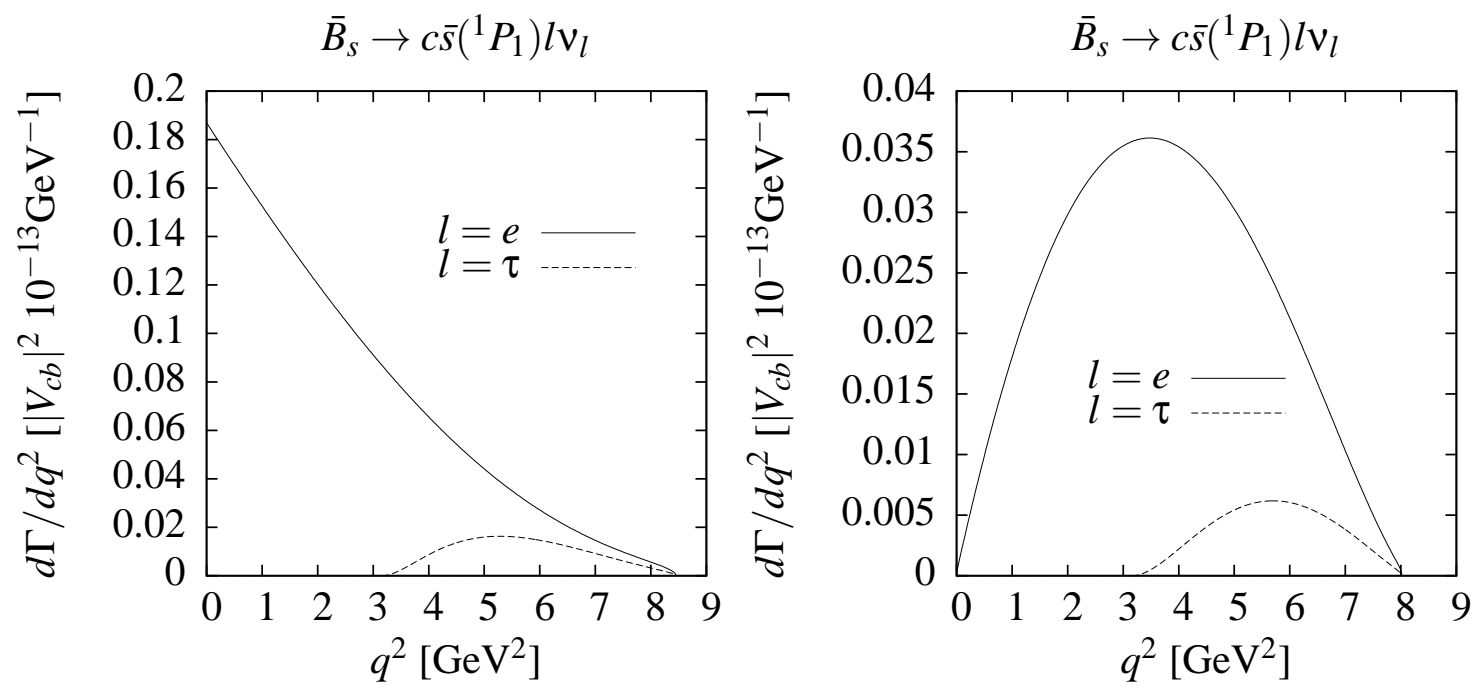

FIG. 7. Differential decay widths for the semileptonic decays of $\bar{B}_{S}$ into $J^{P}=1^{+}, S=0$ (left panel) and $J^{P}=1^{+}, S=1$ (right panel) states.
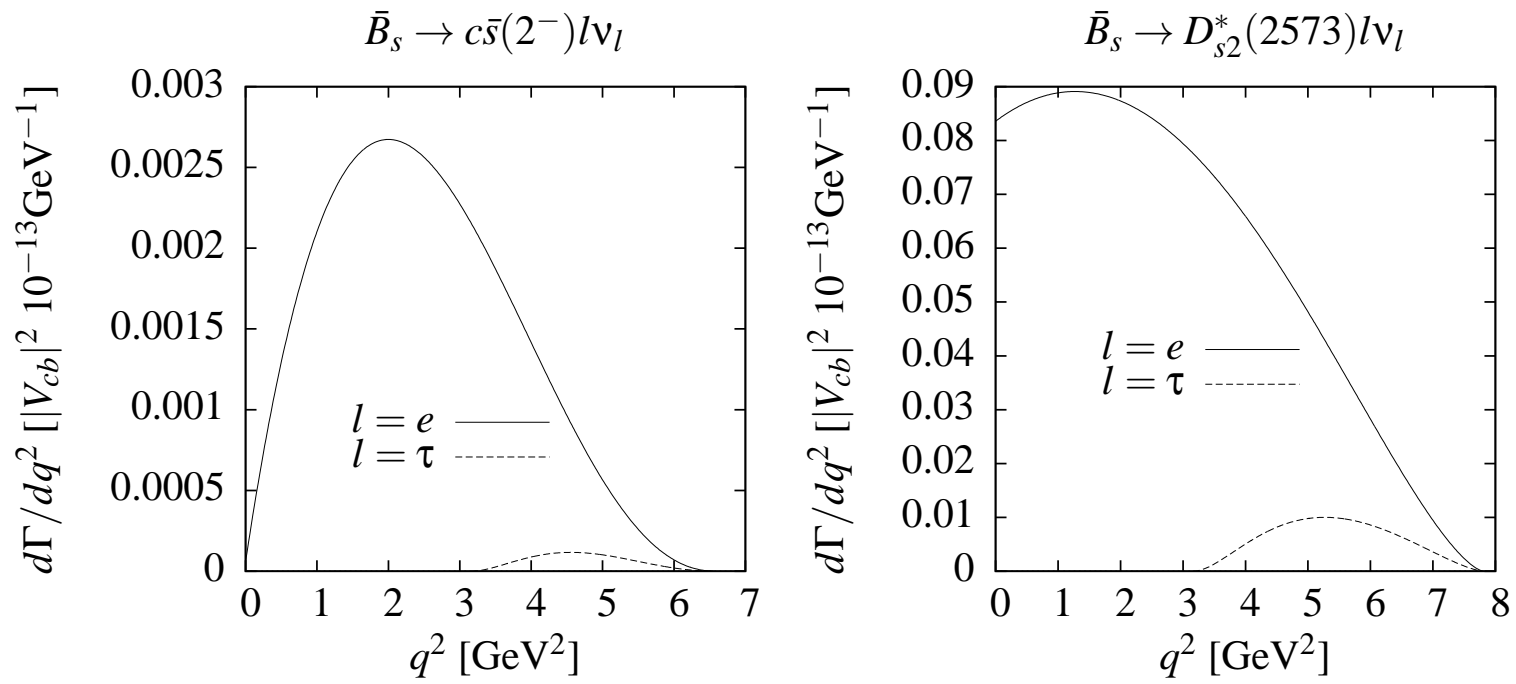

FIG. 8. Differential decay widths for the semileptonic decays of $\bar{B}_{s}$ into $J^{P}=2^{-}$(left panel) and $J^{P}=2^{+}$(right panel) states.

where $r=m_{c \bar{s}} / m_{\bar{B}_{s}}$. These ratios are expected to vary smoothly with $\omega$.

In the case of the semileptonic decays of $B$ mesons, one expects discrepancies of the order of $10-15 \%$ from the predictions of HQET at most. For $B_{s}$ mesons, one should expect, in principle the same kind of unaccuracies than in the $B$ case. Figure 9 shows that this is indeed the case.

At most, $h_{-}$or $h_{a_{2}}$ differ from 0 at the level of the $15 \%$ approximatelly. On the other hand the ratio $R_{1}$ gives an estimate of the discrepancies from the Isgur-Wise function, being these much smaller. These negligible corrections to the heavy quark limit 

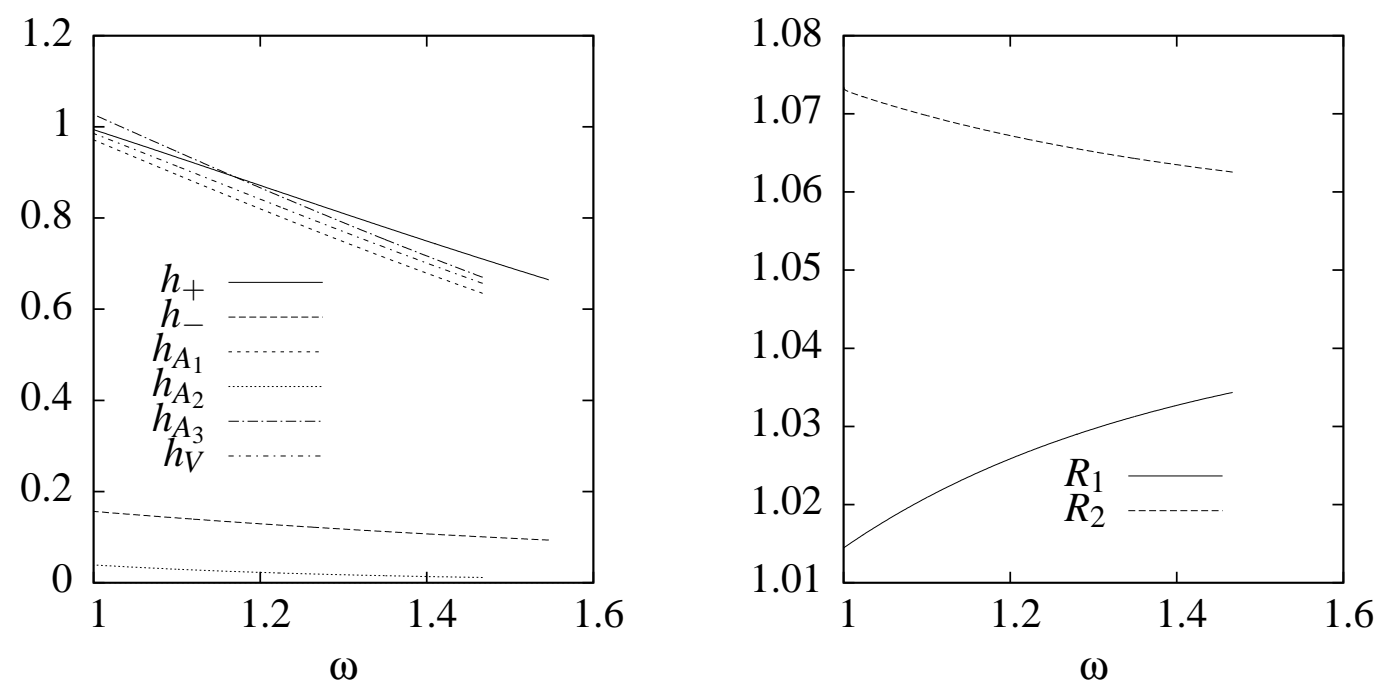

FIG. 9. $h$ form factors for the decay of $\bar{B}_{S}$ mesons into pseudoscalar and vector $c \bar{s}$ states (left panel) and ratios $R_{1}$ and $R_{2}$ (right panel).

predictions in the case of $h_{+}$at $\omega=1$ were also found in the similar calculation carried out in Ref. [23] for the semileptonic $B \rightarrow D$ decay, and this quite small violations might be related to the NRCQM approach. Sum rule and lattice calculation predicts somehow larger corrections, though much smaller than those that affect to $h_{-}$. This is because Luke's theorem, that guaranties that corrections to $h_{+}(1)$ are order $O\left(1 / M_{Q}^{2}\right)$.

\section{SEMILEPTONIC $\bar{B}_{s}$ TO $B^{-}$AND $B^{*-}$ DECAYS}

In principle, one could also consider those weak processes of $B_{s}$ driven by the $\bar{s} \rightarrow \bar{u}$ decays at the quark level. In this case, due to the similar value of the masses of the $\bar{B}_{s}, B$ and $B^{*}$ mesons $\left(m_{\bar{B}_{s}}-m_{B^{-}}=87 \mathrm{MeV}, m_{\bar{B}_{s}}-m_{B^{*-}}=41 \mathrm{MeV}\right)$, the only decay modes allowed are the semileptonic $\bar{B}_{s} \rightarrow B^{-} e^{+} v_{e}$ and $\bar{B}_{s} \rightarrow B^{*-} e^{+} v_{e}$, as the muon, for instance, lay beyond the scope of the available phase space, so that other semileptonic or nonleptonic processes are forbidden.

Let us consider first the $\bar{B}_{s} \rightarrow B^{-}$transition. This process involve a pseudoscalar to pseudoscalar transition, so we take the following form factor decomposition

$$
\left\langle B^{-} \vec{P}_{B^{-}}\left|J_{\mu}^{s u}(0)\right| \bar{B}_{s}, \vec{P}_{\bar{B}_{s}}\right\rangle=P_{\mu} F_{+}\left(q^{2}\right)+q_{\mu} F_{-}\left(q^{2}\right)
$$

The expressions of the form factors are exactly the same of those of Eq.12

The total decay width of this process results to be

$$
\Gamma_{B_{s} \rightarrow B^{-} e^{+} v_{e}}=1.710^{-20} \mathrm{GeV}
$$

For the process $\bar{B}_{s} \rightarrow B^{*-} e^{+} v_{e}$ process. Again, the form factor decomposition is the same as that of the $B_{s} \rightarrow D_{s}^{*}$ decay,

$$
\begin{aligned}
\left\langle B^{*} \lambda \vec{P}_{c \bar{s}}\left|J_{\mu}^{s u}(0)\right| B_{s}, \vec{P}_{B_{s}}\right\rangle & =\frac{-1}{m_{B_{s}}+m_{c \bar{s}}} \varepsilon_{\mu v \alpha \beta} \varepsilon_{(\lambda)}^{v *}\left(\vec{P}_{c \bar{s}}\right) P^{\alpha} q^{\beta} V\left(q^{2}\right) \\
& -i\left\{\left(m_{B_{s}}-m_{c \bar{s}}\right) \varepsilon_{(\lambda) \mu}^{*}\left(\vec{P}_{c \bar{s}}\right) A_{0}\left(q^{2}\right)-\frac{P \cdot \varepsilon_{(\lambda)}^{*}\left(\vec{P}_{c \bar{s}}\right)}{m_{B_{s}}+m_{c \bar{s}}}\left(P_{\mu} A_{+}\left(q^{2}\right)+q_{\mu} A_{-}\left(q^{2}\right)\right)\right\}
\end{aligned}
$$

and the expression of the form factor is that of Eq.16. Now we obtain

$$
\Gamma_{B_{s} \rightarrow B^{*} e^{-} \bar{v}_{e}}=7.610^{-22} \mathrm{GeV}
$$


The decay widths of these transitions are several orders of magnitude smaller than other corresponding to reactions involving a $b \rightarrow c$ transition. One could expect this fact due to the reduced phase space available for reactions driven by a $s \rightarrow u$ transition at the quark level.

\section{NONLEPTONIC $B_{s} \rightarrow c \bar{s} M_{F}$ TWO MESON DECAYS}

In this section we evaluate decay widths for nonleptonic $\bar{B}_{S} \rightarrow c \bar{s} M_{F}$ two-meson decays where $M_{F}$ is a pseudoscalar or vector meson. These decays correspond to a $b \rightarrow c$ transition at the quark level. These transitions are governed, neglecting penguin operators, by the effective Hamiltonian [46, 47]

$$
H_{\mathrm{eff}}=\frac{G_{F}}{\sqrt{2}}\left(V_{c b}\left[c_{1}(\mu) Q_{1}^{c b}+c_{2}(\mu) Q_{2}^{c b}\right]+H . c .\right),
$$

where $c_{1,2}$ are scale-dependent Wilson coefficients, and $Q_{1,2}$ are local four-quark operators given by

$$
\begin{array}{r}
Q_{1}^{c b}=\bar{\Psi}_{c}(0) \gamma_{\mu}\left(I-\gamma_{5}\right) \Psi_{b}(0)\left[V_{u d}^{*} \bar{\Psi}_{d}(0) \gamma^{\mu}\left(I-\gamma_{5}\right) \Psi_{u}(0)+V_{u s}^{*} \bar{\Psi}_{s}(0) \gamma^{\mu}\left(I-\gamma_{5}\right) \Psi_{u}(0)\right. \\
\left.+V_{c d}^{*} \bar{\Psi}_{d}(0) \gamma^{\mu}\left(I-\gamma_{5}\right) \Psi_{c}(0)+V_{c s}^{*} \bar{\Psi}_{s}(0) \gamma^{\mu}\left(I-\gamma_{5}\right) \Psi_{c}(0)\right] \\
Q_{2}^{c b}=\bar{\Psi}_{d}(0) \gamma_{\mu}\left(I-\gamma_{5}\right) \Psi_{b}(0)\left[V_{u d}^{*} \bar{\Psi}_{c}(0) \gamma^{\mu}\left(I-\gamma_{5}\right) \Psi_{u}(0)+V_{c d}^{*} \bar{\Psi}_{c}(0) \gamma^{\mu}\left(I-\gamma_{5}\right) \Psi_{c}(0)\right] \\
+\bar{\Psi}_{s}(0) \gamma_{\mu}\left(I-\gamma_{5}\right) \Psi_{b}(0)\left[V_{u s}^{*} \bar{\Psi}_{c}(0) \gamma^{\mu}\left(I-\gamma_{5}\right) \Psi_{u}(0)+V_{c s}^{*} \bar{\Psi}_{c}(0) \gamma^{\mu}\left(I-\gamma_{5}\right) \Psi_{c}(0)\right]
\end{array}
$$

where $V_{i j}$ are CKM matrix elements. We shall work in the factorization approximation, i. e., the hadron matrix elements of the effective Hamiltonian are evaluated as a product of quark-current matrix elements. One of these is the matrix element of the $B_{s}$ transition to one of the final mesons, while the other corresponds to the transition to the vacuum to the other final mesons, which is given by the corresponding meson decay constant. This is depicted in Fig. 10

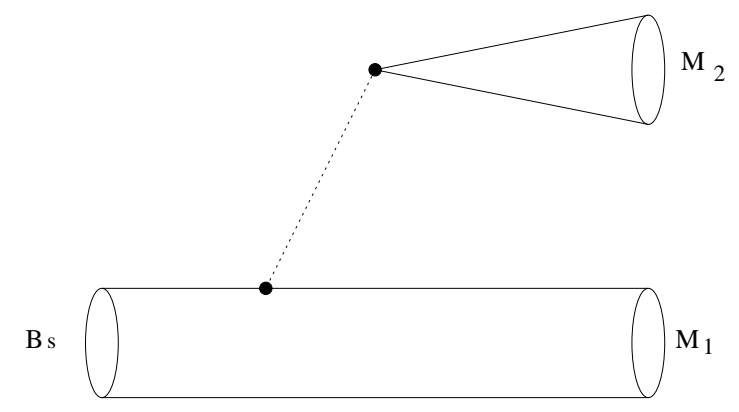

FIG. 10. Diagrammatic representation of $\bar{B}_{S}$ two meson decay in the factorization approximation.

When writing the factorization amplitude one has to take into account the Fierz reordered contribution so that the relevant coefficients are not $c_{1}$ and $c_{2}$, but the combinations

$$
\begin{aligned}
& a_{1}(\mu)=c_{1}(\mu)+\frac{1}{N_{C}} c_{2}(\mu) \\
& a_{2}(\mu)=c_{2}(\mu)+\frac{1}{N_{C}} c_{1}(\mu)
\end{aligned}
$$

with $N_{C}=3$ the number of colors. The appropriate energy scale $(\mu)$ in our case is $\mu \approx m_{b}$, providing the following values for $a_{1,2}[48]$ :

$$
a_{1}=1.14 \quad a_{2}=-0.20 .
$$

$$
\text { A. } M_{F}=\pi, \rho, K, K^{*}
$$

For final states containing one of these mesons, the decay width is given by

$$
\Gamma=\frac{G_{F}^{2}}{16 \pi m_{B_{s}}^{2}}\left|V_{b c}\right|^{2}\left|V_{F}\right|^{2} \frac{\lambda^{1 / 2}\left(m_{B_{s}}^{2}, m_{c \bar{s}}^{2}, m_{M_{F}}\right)^{2}}{2 m_{B_{s}}} a_{1}^{2} \mathcal{H}_{\alpha \beta}\left(P_{B_{s}}, P_{c \bar{s}}\right) \hat{\mathcal{H}}^{\alpha \beta}\left(P_{F}\right),
$$




\begin{tabular}{|c|c|c|c|c|c|}
\hline & $\Gamma\left[10^{-15} \mathrm{GeV}\right]$ & & $\Gamma\left[10^{-15} \mathrm{GeV}\right]$ & & $\Gamma\left[10^{-15} \mathrm{GeV}\right]$ \\
\hline $\bar{B}_{s} \rightarrow D_{s}^{+} \pi^{-}$ & $1.84_{-0.03}^{+0.04} a_{1}^{2}$ & $\bar{B}_{s} \rightarrow D_{s}^{*+} \pi^{-}$ & $1.56_{-0.04}^{+0.1} a_{1}^{2}$ & $\bar{B}_{s} \rightarrow\left(2^{-}\right)^{+} \pi^{-}$ & $2.46_{-0.27}^{+0.09} 10^{-4} a_{1}^{2}$ \\
\hline $\bar{B}_{s} \rightarrow D_{s}^{+} \rho^{-}$ & $4.53_{-0.09}^{+0.1} a_{1}^{2}$ & $\bar{B}_{s} \rightarrow D_{s}^{*+} \rho^{-}$ & $4.67_{-0.11}^{+0.3} a_{1}^{2}$ & $\bar{B}_{s} \rightarrow\left(2^{-}\right)^{+} \rho^{-}$ & $1.62_{-0.26} 10^{-2} a_{1}^{2}$ \\
\hline $\bar{B}_{s} \rightarrow D_{s}^{+} K^{-}$ & $0.14^{+0.01} a_{1}^{2}$ & $\bar{B}_{s} \rightarrow D_{s}^{*+} K^{-}$ & $0.12_{-0.01}^{+0.01} a_{1}^{2}$ & $\bar{B}_{s} \rightarrow\left(2^{-}\right)^{+} K^{-}$ & $1.82_{-0.2}^{+0.06} 10^{-5} a_{1}^{2}$ \\
\hline $\bar{B}_{s} \rightarrow D_{s}^{+} K^{*-}$ & $0.25_{-0.01}^{0.01} a_{1}^{2}$ & $\bar{B}_{s} \rightarrow D_{s}^{*+} K^{*-}$ & $0.27_{-0.01}^{+0.02} a_{1}^{2}$ & $\bar{B}_{s} \rightarrow\left(2^{-}\right)^{+} K^{*-}$ & $1.11_{-0.12} 10^{-3} a_{1}^{2}$ \\
\hline $\bar{B}_{s} \rightarrow D_{s 0}^{*+} \pi^{-}$ & $0.39_{-0.01}^{+0.01} a_{1}^{2}$ & $\bar{B}_{s} \rightarrow D_{s 1}^{+}(2460) \pi^{-}$ & $0.53_{-0.02} a_{1}^{2}$ & $\bar{B}_{s} \rightarrow D_{s 2}^{*+} \pi^{-}$ & $0.35_{-0.03} a_{1}^{2}$ \\
\hline $\bar{B}_{s} \rightarrow D_{s 0}^{*+} \rho^{-}$ & $0.94_{-0.04}^{+0.04} a_{1}^{2}$ & $\bar{B}_{s} \rightarrow D_{s 1}^{+}(2460) \rho^{-}$ & $1.26_{-0.06} a_{1}^{2}$ & $\bar{B}_{s} \rightarrow D_{s 2}^{*+} \rho^{-}$ & $0.95_{-0.07} a_{1}^{2}$ \\
\hline $\bar{B}_{s} \rightarrow D_{s 0}^{*+} K^{-}$ & $2.98_{-0.15}^{+0.11} 10^{-2} a_{1}^{2}$ & $\bar{B}_{s} \rightarrow D_{s 1}^{+}(2460) K^{-}$ & $4.09_{-0.1} 10^{-2} a_{1}^{2}$ & $\bar{B}_{s} \rightarrow D_{s 2}^{*+} K^{-}$ & $2.61_{-0.17} 10^{-2} a_{1}^{2}$ \\
\hline \multirow[t]{5}{*}{$\bar{B}_{s} \rightarrow D_{s 0}^{*+} K^{*-}$} & $5.19_{-0.24}^{+0.18} 10^{-2} a_{1}^{2}$ & $\bar{B}_{s} \rightarrow D_{s 1}^{+}(2460) K^{*-}$ & $6.93_{-0.3} 10^{-2} a_{1}^{2}$ & $\bar{B}_{s} \rightarrow D_{s 2}^{*+} K^{*-}$ & $5.41_{-0.37} 10^{-2} a_{1}^{2}$ \\
\hline & & $\bar{B}_{s} \rightarrow D_{s 1}^{+}(2536) \pi^{-}$ & $0.25_{-0.01} a_{1}^{2}$ & & \\
\hline & & $\bar{B}_{s} \rightarrow D_{s 1}^{+}(2536) \rho^{-}$ & $0.66_{-0.03} a_{1}^{2}$ & & \\
\hline & & $\bar{B}_{s} \rightarrow D_{s 1}^{+}(2536) K^{-}$ & $1.94_{-0.08} 10^{-2} a_{1}^{2}$ & & \\
\hline & & $\bar{B}_{s} \rightarrow D_{s}^{+}(2536) K^{*-}$ & $3.75_{-0.2} 10^{-2} a_{1}^{2}$ & & \\
\hline
\end{tabular}

TABLE VIII. Total nonleptonic decay widths of $B_{s}$ mesons for generic values of the Wilson parameter $a_{1}$. The central values have been calculated using the AL1 potential.

where $m_{f}$ is the mass of the $M_{F}$ meson, $V_{F}$ is $V_{u d}$ for $M_{F}=\pi, \rho$ and $V_{u s}$ for $M_{F}=K, K^{*} . \mathcal{H}_{\alpha \beta}\left(P_{B_{s}}, P_{c \bar{s}}\right)$ is the hadron tensor accounting for the $B_{s} \rightarrow c \bar{s}$ transition, while the other, $\mathcal{H}^{\alpha \beta}\left(P_{F}\right)$ corresponds to a vacuum $\rightarrow M_{F}$ transition. This is equal to

$$
\hat{\mathcal{H}}^{\alpha \beta}\left(P_{F}\right)=p_{F}^{\alpha} p_{F}^{\beta} f_{F}^{2}
$$

for a pseudoscalar $M_{F}$, and

$$
\hat{\mathcal{H}}^{\alpha \beta}\left(P_{F}\right)=\left(p_{F}^{\alpha} p_{F}^{\beta}-m_{F}^{2} g^{\alpha \beta}\right) f_{F}^{2}
$$

for a vector $M_{F}$. All the necessary meson decay constants can be found in Table II

As we did in the case of semileptonic decays, the contraction of the two hadron tensors can be written in terms of helicity amplitudes. For a pseudoscalar $M_{F}$, this is

$$
\mathcal{H}_{\alpha \beta} \tilde{\mathcal{H}}^{\alpha \beta}=\mathcal{H}_{t t}\left(m_{F}^{2}\right) m_{F}^{2} f_{F}^{2},
$$

and for a vector $M_{F}$,

$$
\mathcal{H}_{\alpha \beta} \tilde{\mathcal{H}}^{\alpha \beta}=m_{F}^{2} f_{F}^{2}\left(\mathcal{H}_{1+1}\left(m_{F}^{2}\right)+\mathcal{H}_{1-1}\left(m_{F}^{2}\right)+\mathcal{H}_{00}\left(m_{F}^{2}\right)\right)
$$

In Table VIII we show the values for the decay widths we obtain for the nonleptonic decay widths of the different channels considered in units of $10^{-15} \mathrm{GeV}$. In Table IX we express our results as branching fractions and compare with other calculations. As shown there, our results agree with those from Refs. [28] and [36] in which relativistic CQM and QCD sum rules techniques were used, respectively. Our results for decays with a vector $D_{s}^{*}$ in the final state also agree finely with those from [32], although our values for final states with a pseudoscalar $D_{s}$ meson in the final state are about a factor 2 larger than those from [32], who also works in the context of nonrelativistic constituent quark models. The values calculated in this work are larger by a factor 2 or more than the results from Refs. [33], [49] and [50], in which a light cone sum rules, QCD sum rules and covariant light front quark model approaches have been used. Finally we compare our results with the experimental measurements enclosed in Ref. [39].

$$
\text { B. } M_{F}=D, D_{s}, D^{*}, D_{s}^{*}
$$

In the same way, we can calculate the nonleptonic decay width of the processes $\bar{B}_{s} \rightarrow D_{s} D, \bar{B}_{s} \rightarrow D_{s} D^{*}, \bar{B}_{s} \rightarrow D_{s}^{*} D_{s}, \bar{B}_{s} \rightarrow$ $D_{s}^{*} D_{s}^{*}, \bar{B}_{s} \rightarrow D_{s} D_{s}^{*}$, and $\bar{B}_{s} \rightarrow D_{s}^{*} D_{s}^{*}$ decays. As in the previous case, there is only one contribution proportional to the coefficient $a_{1}$, with momentum transfer ranges between $m_{D}^{2}$ and $m_{D^{*}}^{2}$. These momentum transfers are neither too high (so there is no need to involve a $B_{s}^{*}$ resonance) nor too low (with a high trimomentum transfer). For $M_{F}=D, D^{*}, D_{S}, D_{s}^{*}$, the relevant contractions for the hadron tensors can be obtained from Eqs. 60 and 61, performing straigthforward substitutions. As in the previous case, the decay constants relevant for these calculations can be found in Table 


\begin{tabular}{|c|c|c|c|c|c|c|c|c|}
\hline & This work & [28] & [36] & [32] & [33] & [49] & {$[50]$} & Experiment [39] \\
\hline $\bar{B}_{s} \rightarrow D_{s}^{+} \pi^{-}$ & 0.53 & 0.35 & 0.5 & $0.27_{-0.03}^{+0.07}$ & $0.17_{-0.06}^{+0.07}$ & $0.142 \pm 0.57$ & $0.196_{-0.097}^{+0.123}$ & $0.32 \pm 0.4$ \\
\hline $\bar{B}_{s} \rightarrow D_{s}^{+} \rho^{-}$ & 1.26 & 0.94 & 1.3 & $0.64_{-0.11}^{+0.17}$ & $0.42_{-1.4}^{+1.7}$ & & $0.47_{-2.3}^{+2.9}$ & $0.74 \pm 0.17$ \\
\hline $\bar{B}_{s} \rightarrow D_{s}^{+} K^{-}$ & 0.04 & 0.028 & 0.04 & $0.021_{-0.002}^{+0.002}$ & $0.013_{-0.004}^{+0.005}$ & $0.0103 \pm 0.0051$ & $0.017_{-0.0066}^{+0.0087}$ & \\
\hline $\bar{B}_{S} \rightarrow D_{s}^{+} K^{*-}$ & 0.08 & 0.047 & 0.06 & $0.038_{-0.005}^{+0.005}$ & $0.028_{-0.08}^{+0.01}$ & $0.005 \pm 0.0022$ & & \\
\hline $\bar{B}_{s} \rightarrow D_{s 0}^{*+} \pi^{-}$ & 0.10 & 0.09 & $0.052_{-0.021}^{+0.25}$ & & & & & \\
\hline $\bar{B}_{s} \rightarrow D_{s 0}^{*+} \rho^{-}$ & 0.27 & 0.22 & $0.013_{-0.05}^{+0.06}$ & & & & & \\
\hline $\bar{B}_{s} \rightarrow D_{s 0}^{*+} K^{-}$ & 0.009 & 0.007 & $0.004_{-0.002}^{+0.002}$ & & & & & \\
\hline $\bar{B}_{s} \rightarrow D_{s 0}^{*+} K^{*-}$ & 0.16 & 0.012 & $0.008_{-0.003}^{+0.004}$ & & & & & \\
\hline $\bar{B}_{s} \rightarrow D_{s}^{*+} \pi^{-}$ & 0.45 & 0.27 & 0.2 & $0.31_{-0.02}^{+0.03}$ & & $0.211 \pm 0.073$ & $0.189_{-0.093}^{+0.120}$ & $0.21 \pm 0.06$ \\
\hline $\bar{B}_{s} \rightarrow D_{s}^{*+} \rho^{-}$ & 1.35 & 0.87 & 1.3 & $0.9_{-1.5}^{+1.5}$ & & & $0.523_{-0.256}^{+0.334}$ & $1.03 \pm 2.6$ \\
\hline $\bar{B}_{s} \rightarrow D_{s}^{*+} K^{-}$ & 0.04 & 0.021 & 0.02 & $0.024_{-0.002}^{+0.002}$ & & & & \\
\hline $\bar{B}_{s} \rightarrow D_{s}^{*+} K^{*-}$ & 0.08 & 0.048 & 0.06 & $0.056_{-0.007}^{+0.006}$ & & & & \\
\hline $\bar{B}_{s} \rightarrow D_{s 1}^{+}(2460) \pi^{-}$ & 0.15 & 0.19 & & & & & & \\
\hline $\bar{B}_{s} \rightarrow D_{s 1}^{+}(2460) \rho^{-}$ & 0.36 & 0.49 & & & & & & \\
\hline $\bar{B}_{s} \rightarrow D_{s 1}^{+}(2460) K^{-}$ & 0.012 & 0.014 & & & & & & \\
\hline $\bar{B}_{s} \rightarrow D_{s 1}^{+}(2460) K^{*-}$ & 0.020 & 0.026 & & & & & & \\
\hline $\bar{B}_{s} \rightarrow D_{s 1}^{+}(2536) \pi^{-}$ & 0.07 & 0.029 & & & & & & \\
\hline $\bar{B}_{s} \rightarrow D_{s 1}^{+}(2536) \rho^{-}$ & 0.19 & 0.083 & & & & & & \\
\hline $\bar{B}_{s} \rightarrow D_{s 1}^{+}(2536) K^{-}$ & 0.0054 & 0.0021 & & & & & & \\
\hline $\bar{B}_{s} \rightarrow D_{s 1}^{+}(2536) K^{*-}$ & 0.01 & 0.0044 & & & & & & \\
\hline $\bar{B}_{S} \rightarrow\left(2^{-}\right)^{+} \pi^{-}$ & $7.110^{-5}$ & & & & & & & \\
\hline $\bar{B}_{s} \rightarrow\left(2^{-}\right)^{+} \rho^{-}$ & 0.0047 & & & & & & & \\
\hline $\bar{B}_{s} \rightarrow\left(2^{-}\right)^{+} K^{-}$ & $5.210^{-6}$ & & & & & & & \\
\hline $\bar{B}_{s} \rightarrow\left(2^{-}\right)^{+} K^{*-}$ & $2.210^{-8}$ & & & & & & & \\
\hline $\bar{B}_{s} \rightarrow D_{s 2}^{*+} \pi^{-}$ & 0.1 & 0.16 & & & & & & \\
\hline $\bar{B}_{s} \rightarrow D_{s 2}^{*+} \rho^{-}$ & 0.27 & 0.42 & & & & & & \\
\hline $\bar{B}_{s} \rightarrow D_{s 2}^{*+} K^{-}$ & 0.008 & 0.012 & & & & & & \\
\hline $\bar{B}_{s} \rightarrow D_{s 2}^{*+} K^{*-}$ & 0.016 & 0.022 & & & & & & \\
\hline
\end{tabular}

TABLE IX. Branching ratios for the decays above.

\begin{tabular}{lc}
\hline \hline & $\Gamma\left[10^{-15} \mathrm{GeV}\right]$ \\
\hline $\bar{B}_{s} \rightarrow D_{s}^{+} D_{s}^{-}$ & $7.35_{-0.14}^{+0.04} a_{1}^{2}$ \\
$\bar{B}_{s} \rightarrow D_{s}^{+} D_{s}^{*-}$ & $6.89_{-0.13}^{+0.02} a_{1}^{2}$ \\
$\bar{B}_{s} \rightarrow D_{s}^{*+} D_{s}^{-}$ & $4.23_{-0.38} a_{1}^{2}$ \\
$\bar{B}_{s} \rightarrow D_{s}^{*+} D_{s}^{*-}$ & $18.79_{-1.6} a_{1}^{2}$ \\
\hline $\bar{B}_{s} \rightarrow D_{s}^{+} D^{-}$ & $0.25_{-0.01} a_{1}^{2}$ \\
$\bar{B}_{s} \rightarrow D_{s}^{+} D^{*-}$ & $0.19_{-0.01} a_{1}^{2}$ \\
$\bar{B}_{s} \rightarrow D_{s}^{*+} D^{-}$ & $0.15_{-0.01}^{+0.01} a_{1}^{2}$ \\
$\bar{B}_{s} \rightarrow D_{s}^{*+} D^{*-}$ & $0.46_{-0.01}^{+0.02} a_{1}^{2}$ \\
\hline
\end{tabular}

TABLE X. Nonleptonic decay widths for the indicated processes indicated, using the factorization approximation. We give our results for generic values of the parameter $a_{1}$.

Results are enclosed in Table $\mathrm{X}$ In Table $\mathrm{XI}$, we present our values as branching fractions, and compare with other results, in the case of decays with two $D_{s}^{(*)}$ mesons in the final state. We have found a fair agreement with the branching fractions calculated in [28] and [36], and larger differences with the values given in [32], [33] and [51]. Most of the values calculated here and those found in the literature differ from the experimental results by a factor around 2. Appart from the inaccuracies of the factorization approximation, the results are sensitive, not only two the Wilson parameter $a_{1}$, also on the value that have been used for the mesons decay constant and on the overlap among the wave functions used to calculate the matrix elements. 


\begin{tabular}{l|cccccc|c}
\hline \hline & This work [28] $[36]$ & {$[32]$} & {$[33]$} & {$[$ [51] } & Experiment [39] \\
\hline $\bar{B}_{s} \rightarrow D_{s}^{+} D_{s}^{-}$ & 2.1 & 1.1 & 1.0 & $0.83_{-0.1}^{+0.1}$ & 1.65 & $0.217 \pm 0.082$ & $0.53 \pm 0.09$ \\
$\bar{B}_{s} \rightarrow D_{s}^{+} D_{s}^{*-}$ & 2.0 & 1.0 & 0.8 & $0.84_{-0.12}^{+0.12}$ & $0.262 \pm 0.93$ & \\
$\bar{B}_{s} \rightarrow D_{s}^{*+} D_{s}^{-}$ & 1.24 & 0.61 & 0.4 & $0.7_{-0.15}^{+1.6}$ & $0.254 \pm 0.57$ & \\
$\bar{B}_{s} \rightarrow D_{s}^{+} D_{s}^{*-}+D_{s}^{*+} D_{s}^{-}$ & 3.24 & 1.61 & 1.2 & $1.54_{-0.19}^{+0.2}$ & 2.4 & $5.16 \pm 0.11$ & $1.24 \pm 0.21$ \\
$\bar{B}_{s} \rightarrow D_{s}^{*+} D_{s}^{*-}$ & 5.45 & 2.5 & 1.6 & $2.4_{-0.4}^{+0.4}$ & 3.18 & $2.77 \pm 0.76$ & $1.88 \pm 0.34$ \\
$\bar{B}_{s} \rightarrow D_{s}^{(*)+} D_{s}^{(*)-}$ & 10.8 & 5.21 & 3.8 & $4.77_{-0.46}^{+0.46}$ & 7.23 & $3.5 \pm 0.78$ & $4.5 \pm 1.4$ \\
\hline $\bar{B}_{s} \rightarrow D_{s}^{+} D^{-}$ & 0.08 & & & & & & \\
$\bar{B}_{s} \rightarrow D_{s}^{+} D^{*-}$ & 0.05 & & & & & & \\
$\bar{B}_{s} \rightarrow D_{s}^{*+} D^{-}$ & 0.04 & 0.13 & & & & & \\
$\bar{B}_{s} \rightarrow D_{s}^{*+} D^{*-}$ & 0.13 & & \\
\hline
\end{tabular}

TABLE XI. Branching ratios in \% for the decays indicated above. We also compare with other calculations.

\begin{tabular}{lcc|c}
\hline \hline & $\Gamma\left[10^{-15} \mathrm{GeV}\right]$ & BR in $\%$ & Experiment [39] \\
\hline $\bar{B}_{S} \rightarrow \phi J / \Psi$ & $11.80_{-0.8}^{+1.9} a_{2}^{2}$ & 0.11 & $\left(0.109_{-0.23}^{+0.28}\right)$ \\
$\bar{B}_{S} \rightarrow K^{0} J / \Psi$ & $8.1_{-1.3}^{+0.5} 10^{-2} a_{2}^{2}$ & $7.2510^{-4}$ & $(3.6 \pm 0.8) 10^{-3}$ \\
$\bar{B}_{S} \rightarrow K^{* 0} J / \Psi$ & $0.51_{-0.3} a_{2}^{2}$ & $4.610^{-3}$ & $(9 \pm 4) 10^{-3}$ \\
\hline
\end{tabular}

TABLE XII. Branching ratios in \% for the reactions indicated above. We give our results for generic values of $a_{2}$.

\section{OTHER NONLEPTONIC DECAYS}

The calculation of decay channels

$$
\begin{aligned}
& \bar{B}_{s} \rightarrow \phi J / \Psi \\
& \bar{B}_{s} \rightarrow K^{0} J / \Psi \\
& \bar{B}_{s} \rightarrow K^{* 0} J / \Psi
\end{aligned}
$$

in the factorization approximation can be easily performed. Their decay width are summarized in Table XII

In Table XII we also give the branching fractions of these channels in $\%$, for generic values of the Wilson parameter $a_{2}$, and compare with the experimental measurements. Our result for the branching ratio corresponding to the decay into $K^{0} J / \Psi$ states reproduces roughly the order of magnitude of the corresponding experimental value $\left(\approx 10^{-3}\right)$. In contrast, our results for the branching fractions for the $\bar{B}_{s} \rightarrow \phi J / \Psi$ and $\bar{B}_{s} \rightarrow K^{* 0} J / \Psi$ decays agree with the experimental data of Ref. [39].

\section{SUMMARY AND CONCLUSIONS}

In this paper we have studied the semileptonic decays of the $\bar{B}_{s}$ meson into $c \bar{s}$ states with $J^{P}=0^{-}, 0^{+}, 1^{-}, 1^{+}, 2^{-}$and $2^{+}$. We have worked in the context of nonrelativistic constituent quark models. We compare with the experimental results enclosed in Ref. [39] when possible. We have also computed several nonleptonic decay modes of $\bar{B}_{s}$ mesons. We work in the factorization approximation, as the momenta involved does not involve resonances or high trimomentum transfer. We give results for general values of the Wilson coefficients. We give an estimate of our theoretical uncertainties by considering different sets of wave functions derived from the quark-antiquark potentials of Ref. [42]. The results that we obtain for the semileptonic decay width are in general in good agreement with previous calculations and with the available experimental measurements. In the case of the nonleptonic decay channels that we have studied, we have found reasonable agreement with previous calculations. The nonleptonic decays of $\bar{B}_{s}$ mesons into $\phi J / \Psi, K^{0} J / \Psi$ and $K^{* 0}(892) J / \Psi$ have been considered in this work, finding a good agreement with the experimental results. 


\section{ACKNOWLEDGMENTS}

The author thanks J. Nieves, E. Hernández, M. Á. Pérez-García, I. Vidaña and S. Chiacchiera for useful discussions and kind hospitality. The author also thanks the Physics Department at the University of Coimbra and Departamento de Física Fundamental at University of Salamanca for their hospitality. The author acknowledges a contract from the CPAN project and support from Junta de Andaluca under contract FQM-225. 


\section{Appendix A: Expressions for the matrix elements}

- Case $J^{\pi}=0^{-}$

$$
\begin{aligned}
& V^{0}(|\vec{q}|)=\sqrt{2 m_{I} 2 E_{F}(-\vec{q})} \int d^{3} p \frac{1}{4 \pi}\left(\hat{\phi}_{f_{1}^{\prime}, f_{2}}^{\left(M_{F}\left(0^{-}\right)\right)}(|\vec{p}|)\right)^{*} \hat{\phi}_{f_{1}, f_{2}}^{\left(M_{l}\left(0^{-}\right)\right)}\left(\left|\vec{p}-\frac{m_{f_{2}}}{m_{f_{1}^{\prime}}+m_{f_{2}}}\right| \vec{q}|\vec{k}|\right) \\
& \sqrt{\frac{\widehat{E}_{f_{1}^{\prime}} \widehat{E}_{f_{1}}}{4 E_{f_{1}^{\prime}} E_{f_{1}}}}\left(1+\frac{\left(-\frac{m_{f_{1}^{\prime}}}{m_{f_{1}^{\prime}}+m_{f_{2}}}|\vec{q}| \vec{k}-\vec{p}\right) \cdot\left(\frac{m_{f_{2}}}{m_{f_{1}^{\prime}}}\left|\overrightarrow{m_{f_{2}}}\right| \vec{q} \mid \vec{k}-\vec{p}\right)}{\widehat{E}_{f_{1}^{\prime}} \widehat{f}_{f_{1}}}\right) \\
& V^{3}(|\vec{q}|)=\sqrt{2 m_{I} 2 E_{F}(-\vec{q})} \int d^{3} p \frac{1}{4 \pi}\left(\hat{\phi}_{f_{1}^{\prime}, f_{2}}^{\left(M_{F_{2}}\left(0^{-}\right)\right)}(|\vec{p}|)\right)^{*} \hat{\phi}_{f_{1}, f_{2}}^{\left(M_{l}\left(0^{-}\right)\right)}\left(\left|\vec{p}-\frac{m_{f_{2}}}{m_{f_{1}^{\prime}}+m_{f_{2}}}\right| \vec{q}|\vec{k}|\right) \\
& \sqrt{\frac{\widehat{E}_{f_{1}^{\prime}} \widehat{E}_{f 1}}{4 E_{f_{1}^{\prime}} E_{f_{1}}}}\left(\frac{\frac{m_{f_{2}}}{m_{f_{1}}+m_{f_{2}}}|\vec{q}|-p_{z}}{\widehat{E}_{f_{1}}}+\frac{-\frac{m_{f_{1}^{\prime}}}{m_{f_{1}^{\prime}}+m_{f_{2}}}|\vec{q}|-p_{z}}{\widehat{E}_{f_{1}^{\prime}}}\right)
\end{aligned}
$$

- Case $J^{\pi}=0^{+}$

$$
\begin{aligned}
& A^{0}(|\vec{q}|)=\sqrt{2 m_{I} 2 E_{F}(-\vec{q})} \int d^{3} p \frac{1}{4 \pi|\vec{p}|}\left(\hat{\phi}_{f_{1}^{\prime}, f_{2}}^{\left(M_{F}\left(0^{+}\right)\right)}(|\vec{p}|)\right)^{*} \hat{\phi}_{f_{1}, f_{2}}^{\left(M_{I}\left(0^{-}\right)\right)}\left(\left|\vec{p}-\frac{m_{f_{2}}}{m_{f_{1}^{\prime}}+m_{f_{2}}}\right| \vec{q}|\vec{k}|\right) \\
& \sqrt{\frac{\widehat{E}_{f_{1}^{\prime}} \widehat{E}_{f_{1}}}{4 E_{f_{1}^{\prime}} E_{f_{1}}}}\left(\frac{\vec{p} \cdot\left(\frac{m_{f_{2}}}{m_{f_{1}^{\prime}}+m_{f_{2}}}|\vec{q}| \vec{k}-\vec{p}\right)}{\widehat{E}_{f_{1}}}+\frac{\vec{p} \cdot\left(-\frac{m_{f_{1}^{\prime}}}{m_{f_{1}^{\prime}}+m_{f_{2}}}|\vec{q}| \vec{k}-\vec{p}\right)}{\widehat{E}_{f_{1}^{\prime}}}\right) \\
& A^{3}(|\vec{q}|)=\sqrt{2 m_{I} 2 E_{F}(-\vec{q})} \int d^{3} p \frac{1}{4 \pi|\vec{p}|}\left(\hat{\phi}_{f_{1}^{\prime}, f_{2}}^{\left(M_{F}\left(0^{+}\right)\right)}(|\vec{p}|)\right)^{*} \hat{\phi}_{f_{1}, f_{2}}^{\left(M_{I}\left(0^{-}\right)\right)}\left(\left|\vec{p}-\frac{m_{f_{2}}}{m_{f_{1}^{\prime}}+m_{f_{2}}}\right| \vec{q}|\vec{k}|\right) \\
& \sqrt{\frac{\widehat{E}_{f_{1}^{\prime}} \widehat{E}_{f 1}}{4 E_{f_{1}^{\prime}} E_{f_{1}}}}\left\{p_{z}\left(1-\frac{\left(-\frac{m_{f_{1}^{\prime}}}{m_{f_{1}^{\prime}}+m_{f_{2}}}|\vec{q}| \vec{k}-\vec{p}\right) \cdot\left(\frac{m_{f_{f^{\prime}}}}{m_{f_{1}^{\prime}}}\left|\vec{m} m_{f_{2}}\right| \vec{q} \mid \vec{p}-\vec{p}\right)}{\widehat{E}_{f_{1}^{\prime}} E_{f_{1}}}\right)\right. \\
& +\frac{1}{\widehat{E}_{f_{1}^{\prime}} \widehat{E}_{f 1}}\left[\left(-\frac{m_{f_{1}^{\prime}}}{m_{f_{1}^{\prime}}+m_{f_{2}}}|\vec{q}|-p_{z}\right) \vec{p} \cdot\left(\frac{m_{f_{2}}}{m_{f_{1}^{\prime}}+m_{f_{2}}}|\vec{q}| \vec{k}-\vec{p}\right)\right. \\
& \left.\left.+\left(\frac{m_{f_{2}}}{m_{f_{1}^{\prime}}+m_{f_{2}}}|\vec{q}|-p_{z}\right) \vec{p} \cdot\left(-\frac{m_{f_{1}^{\prime}}}{m_{f_{1}^{\prime}}+m_{f_{2}}}|\vec{q}| \vec{k}-\vec{p}\right)\right]\right\}
\end{aligned}
$$

- Case $J^{\pi}=1^{-}$

$$
\begin{aligned}
& V_{\lambda=-1}^{\left(1^{-}\right) 1}(|\vec{q}|)=\frac{-i}{\sqrt{2}} \sqrt{2 m_{I} 2 E_{F}(-\vec{q})} \int d^{3} p \frac{1}{4 \pi}\left(\hat{\phi}_{f_{1}^{\prime}, f_{2}}^{\left(M_{F}\left(1^{-}\right)\right)}(|\vec{p}|)\right)^{*} \hat{\phi}_{f_{1}, f_{2}}^{\left(M_{I}\left(0^{-}\right)\right)}\left(\left|\vec{p}-\frac{m_{f_{2}}}{m_{f_{1}^{\prime}}+m_{f_{2}}}\right| \vec{q}|\vec{k}|\right) \\
& \sqrt{\frac{\widehat{E}_{f_{1}^{\prime}} \widehat{E}_{f 1}}{4 E_{f_{1}^{\prime}} E_{f_{1}}}}\left(-\frac{\frac{m_{f_{2}}}{m_{f_{1}^{\prime}}+m_{f_{2}}}|\vec{q}|-p_{z}}{\widehat{E}_{f_{1}}}+\frac{-\frac{m_{f_{1}^{\prime}}}{m_{f_{1}^{\prime}}+m_{f_{2}}}|\vec{q}|-p_{z}}{\widehat{E}_{f_{1}^{\prime}}}\right)
\end{aligned}
$$




$$
\begin{aligned}
& A_{\lambda=0}^{\left(1^{-}\right) 0}(|\vec{q}|)=i \sqrt{2 m_{I} 2 E_{F}(-\vec{q})} \int d^{3} p \frac{1}{4 \pi}\left(\hat{\phi}_{f_{1}^{\prime}, f_{2}}^{\left(M_{F}\left(1^{-}\right)\right)}(|\vec{p}|)\right)^{*} \hat{\phi}_{f_{1}, f_{2}}^{\left(M_{I}\left(0^{-}\right)\right)}\left(\left|\vec{p}-\frac{m_{f_{2}}}{m_{f_{1}^{\prime}}+m_{f_{2}}}\right| \vec{q}|\vec{k}|\right) \\
& \sqrt{\frac{\widehat{E}_{f_{1}^{\prime}} \widehat{E}_{f 1}}{4 E_{f_{1}^{\prime}} E_{f_{1}}}}\left(\frac{\frac{m_{f_{2}}}{m_{f_{1}^{\prime}}+m_{f_{2}}}|\vec{q}|-p_{z}}{\widehat{E}_{f_{1}}}+\frac{-\frac{m_{f_{1}^{\prime}}}{m_{f_{1}^{\prime}}+m_{f_{2}}}|\vec{q}|-p_{z}}{\widehat{E}_{f_{1}^{\prime}}}\right) \\
& A_{\lambda=-1}^{\left(1^{-}\right) 1}(|\vec{q}|)=\frac{i}{\sqrt{2}} \sqrt{2 m_{I} 2 E_{F}(-\vec{q})} \int d^{3} p \frac{1}{4 \pi}\left(\hat{\phi}_{f_{1}^{\prime}, f_{2}}^{\left(M_{F}\left(1^{-}\right)\right)}(|\vec{p}|)\right)^{*} \hat{\phi}_{f_{1}, f_{2}}^{\left(M_{I}\left(0^{-}\right)\right)}\left(\left|\vec{p}-\frac{m_{f_{2}}}{m_{f_{1}^{\prime}}+m_{f_{2}}}\right| \vec{q}|\vec{k}|\right) \\
& \sqrt{\frac{\widehat{E}_{f_{1}^{\prime}} \widehat{E}_{f 1}}{4 E_{f_{1}^{\prime}} E_{f_{1}}}}\left(1+\frac{2 p_{x}^{2}-\left(-\frac{m_{f_{1}^{\prime}}}{m_{f_{1}^{\prime}}+m_{f_{2}}}|\vec{q}| \vec{k}-\vec{p}\right) \cdot\left(\frac{m_{f_{2}}}{m_{f_{1}^{\prime}}+m_{f_{2}}}|\vec{q}| \vec{k}-\vec{p}\right)}{\widehat{E}_{f_{1}^{\prime}} \widehat{E}_{f_{1}}}\right) \\
& A_{\lambda=0}^{\left(1^{-}\right) 3}(|\vec{q}|)=i \sqrt{2 m_{I} 2 E_{F}(-\vec{q})} \int d^{3} p \frac{1}{4 \pi}\left(\hat{\phi}_{f_{1}^{\prime}, f_{2}}^{\left(M_{F}\left(1^{-}\right)\right)}(|\vec{p}|)\right)^{*} \hat{\phi}_{f_{1}, f_{2}}^{\left(M_{I}\left(0^{-}\right)\right)}\left(\left|\vec{p}-\frac{m_{f_{2}}}{m_{f_{1}^{\prime}}+m_{f_{2}}}\right| \vec{q}|\vec{k}|\right) \\
& \sqrt{\frac{\widehat{E}_{f_{1}^{\prime}} \widehat{E}_{f 1}}{4 E_{f_{1}^{\prime}} E_{f_{1}}}}\left(1+\frac{2\left(-\frac{m_{f_{1}^{\prime}}}{m_{f_{1}^{\prime}}+m_{f_{2}}}|\vec{q}|-p_{z}\right) \cdot\left(\frac{m_{f_{2}}}{m_{f_{1}^{\prime}}+m_{f_{2}}}|\vec{q}|-p_{z}\right)}{\widehat{E}_{f_{1}^{\prime}} \widehat{E}_{f_{1}}}\right. \\
& \left.-\frac{\left(-\frac{m_{f_{1}^{\prime}}}{m_{f_{1}^{\prime}}+m_{f_{2}}}|\vec{q}| \vec{k}-\vec{p}\right) \cdot\left(\frac{m_{f_{2}}}{m_{f_{1}^{\prime}}+m_{f_{2}}}|\vec{q}| \vec{k}-\vec{p}\right)}{\widehat{E}_{f_{1}^{\prime}} \widehat{E}_{f_{1}}}\right)
\end{aligned}
$$

- Case $J^{\pi}=1^{+}$

$$
\begin{aligned}
& V_{\lambda=0}^{\left(1^{+}, S_{q \bar{q}}=0\right) 0}(|\vec{q}|)=i \sqrt{3} \sqrt{2 m_{I} 2 E_{F}(-\vec{q})} \int d^{3} p \frac{1}{4 \pi|\vec{p}|}\left(\hat{\phi}_{f_{1}^{\prime}, f_{2}}^{\left(M_{F}\left(1^{+}, S_{q \bar{q}}=0\right)\right)}(|\vec{p}|)\right)^{*} \hat{\phi}_{f_{1}, f_{2}}^{\left(M_{I}\left(0^{-}\right)\right)}\left(\left|\vec{p}-\frac{m_{f_{2}}}{m_{f_{1}^{\prime}}+m_{f_{2}}}\right| \vec{q}|\vec{k}|\right) \\
& \sqrt{\frac{\widehat{E}_{f_{1}^{\prime}} \widehat{E}_{f 1}}{4 E_{f_{1}^{\prime}} E_{f_{1}}}} p_{z}\left(1+\frac{\left(-\frac{m_{f_{1}^{\prime}}}{m_{f_{1}^{\prime}}+m_{f_{2}}}|\vec{q}| \vec{k}-\vec{p}\right) \cdot\left(\frac{m_{f_{2}}}{m_{f_{1}^{\prime}}+m_{f_{2}}}|\vec{q}| \vec{k}-\vec{p}\right)}{\widehat{E}_{f_{1}^{\prime}} \widehat{E}_{f_{1}}}\right) \\
& V_{\lambda=0}^{\left(1^{+}, S_{q \bar{q}}=1\right) 0}(|\vec{q}|)=-i \sqrt{\frac{3}{2}} \sqrt{2 m_{I} 2 E_{F}(-\vec{q})} \int d^{3} p \frac{1}{4 \pi|\vec{p}|}\left(\hat{\phi}_{f_{1}^{\prime}, f_{2}}^{\left(M_{F}\left(1^{+}, S_{q \bar{q}}=1\right)\right)}(|\vec{p}|)\right)^{*} \hat{\phi}_{f_{1}, f_{2}}^{\left(M_{I}\left(0^{-}\right)\right)}\left(\left|\vec{p}-\frac{m_{f_{2}}}{m_{f_{1}^{\prime}}+m_{f_{2}}}\right| \vec{q}|\vec{k}|\right) \\
& \sqrt{\frac{\widehat{E}_{f_{1}^{\prime}} \widehat{E}_{f 1}}{4 E_{f_{1}^{\prime}} E_{f_{1}}}} \frac{|\vec{q}|\left(p_{z}^{2}-\vec{p}^{2}\right)}{\widehat{E}_{f_{1}^{\prime}} \widehat{E}_{f 1}} \\
& V_{\lambda=-1}^{\left(1^{+}, S_{q \bar{q}}=0\right) 1}(|\vec{q}|)=-i \sqrt{\frac{3}{2}} \sqrt{2 m_{I} 2 E_{F}(-\vec{q})} \int d^{3} p \frac{1}{4 \pi|\vec{p}|}\left(\hat{\phi}_{f_{1}^{\prime}, f_{2}}^{\left(M_{T}\left(1^{+}, S_{q \bar{q}}=0\right)\right)}(|\vec{p}|)\right)^{*} \hat{\phi}_{f_{1}, f_{2}}^{\left(M_{I}\left(0^{-}\right)\right)}\left(\left|\vec{p}-\frac{m_{f_{2}}}{m_{f_{1}^{\prime}}+m_{f_{2}}}\right| \vec{q}|\vec{k}|\right) \\
& \sqrt{\frac{\widehat{E}_{f_{1}^{\prime}} \widehat{E}_{f 1}}{4 E_{f_{1}^{\prime}} E_{f_{1}}}} p_{x}^{2}\left(\frac{1}{\widehat{E}_{f_{1}}}+\frac{1}{\widehat{E}_{f_{1}^{\prime}}}\right) \\
& V_{\lambda=-1}^{\left(1^{+}, S_{q \bar{q}}=1\right) 1}(|\vec{q}|)=i \frac{\sqrt{3}}{2} \sqrt{2 m_{I} 2 E_{F}(-\vec{q})} \int d^{3} p \frac{1}{4 \pi|\vec{p}|}\left(\hat{\phi}_{f_{1}^{\prime}, f_{2}}^{\left(M_{F}\left(1^{+}, S_{q \bar{q}}=1\right)\right)}(|\vec{p}|)\right)^{*} \hat{\phi}_{f_{1}, f_{2}}^{\left(M_{I}\left(0^{-}\right)\right)}\left(\left|\vec{p}-\frac{m_{f_{2}}}{m_{f_{1}^{\prime}}+m_{f_{2}}}\right| \vec{q}|\vec{k}|\right) \\
& \sqrt{\frac{\widehat{E}_{f_{1}^{\prime}} \widehat{E}_{f 1}}{4 E_{f_{1}^{\prime}} E_{f_{1}}}}\left(\frac{p_{y}^{2}+p_{z}^{2}+p_{z}|\vec{q}| \frac{m_{f_{1}^{\prime}}}{m_{f_{1}^{\prime}}+m_{f_{2}}}}{\widehat{E}_{f_{1}^{\prime}}}-\frac{p_{y}^{2}+p_{z}^{2}-p_{z}|\vec{q}| \frac{m_{f_{2}}}{m_{f_{1}^{\prime}}+m_{f_{2}}}}{\widehat{E}_{f_{1}}}\right)
\end{aligned}
$$




$$
\begin{aligned}
& V_{\lambda=0}^{\left(1^{+}, S_{q} \bar{q}=0\right) 3}(|\vec{q}|)=i \sqrt{3} \sqrt{2 m_{I} 2 E_{F}(-\vec{q})} \int d^{3} p \frac{1}{4 \pi|\vec{p}|}\left(\hat{\phi}_{f_{1}^{\prime}, f_{2}}^{\left(M_{F}\left(1^{+}, S_{q \bar{q}}=0\right)\right)}(|\vec{p}|)\right)^{*} \hat{\phi}_{f_{1}, f_{2}}^{\left(M_{I}\left(0^{-}\right)\right)}\left(\left|\vec{p}-\frac{m_{f_{2}}}{m_{f_{1}^{\prime}}+m_{f_{2}}}\right| \vec{q}|\vec{k}|\right) \\
& \sqrt{\frac{\widehat{E}_{f_{1}^{\prime}} \widehat{E}_{f 1}}{4 E_{f_{1}^{\prime}} E_{f_{1}}}} p_{z}\left(\frac{\frac{m_{f_{2}}}{m_{f_{1}^{\prime}}+m_{f_{2}}}|\vec{q}|-p_{z}}{\widehat{E}_{f_{1}}}+\frac{-\frac{m_{f^{\prime}}}{m_{f_{1}^{\prime}}+m_{f_{2}}}|\vec{q}|-p_{z}}{\widehat{E}_{f_{1}^{\prime}}}\right) \\
& V_{\lambda=0}^{\left(1^{+}, S_{q q}=1\right) 3}(|\vec{q}|)=-i \sqrt{\frac{3}{2}} \sqrt{2 m_{I} 2 E_{F}(-\vec{q})} \int d^{3} p \frac{1}{4 \pi|\vec{p}|}\left(\hat{\phi}_{f_{1}^{\prime}, f_{2}}^{\left(M_{F}\left(1^{+}, S_{q}=1\right)\right)}(|\vec{p}|)\right)^{*} \hat{\phi}_{f_{1}, f_{2}}^{\left(M_{l}\left(0^{-}\right)\right)}\left(\left|\vec{p}-\frac{m_{f_{2}}}{m_{f_{1}^{\prime}}+m_{f_{2}}}\right| \vec{q}|\vec{k}|\right) \\
& \sqrt{\frac{\widehat{E}_{f_{1}} \widehat{E}_{f 1}}{4 E_{f_{1}^{\prime}} E_{f_{1}}}}\left(p_{x}^{2}+p_{y}^{2}\right)\left(\frac{1}{\widehat{E}_{f_{1}}}-\frac{1}{\widehat{E}_{f_{1}^{\prime}}}\right) \\
& A_{\lambda=-1}^{\left(1^{+}, S_{q}=0\right) 1}(|\vec{q}|)=-i \sqrt{\frac{3}{2}} \sqrt{2 m_{I} 2 E_{F}(-\vec{q})} \int d^{3} p \frac{1}{4 \pi|\vec{p}|}\left(\hat{\phi}_{f_{1}^{\prime}, f_{2}}^{\left(M_{F}\left(1^{+}, S_{q}=0\right)\right)}(|\vec{p}|)\right)^{*} \hat{\phi}_{f_{1}, f_{2}}^{\left(M_{l}\left(0^{-}\right)\right)}\left(\left|\vec{p}-\frac{m_{f_{2}}}{m_{f_{1}^{\prime}}+m_{f_{2}}}\right| \vec{q}|\vec{k}|\right) \\
& \sqrt{\frac{\widehat{E}_{f_{1}} \widehat{E}_{f 1}}{4 E_{f_{1}^{\prime}} E_{f_{1}}}} \frac{p_{y}^{2}|\vec{q}|}{\widehat{E}_{f_{1}} \widehat{E}_{f_{1}^{\prime}}} \\
& A_{\lambda=-1}^{\left(1^{+}, S_{q}=1\right) 1}(|\vec{q}|)=i \frac{\sqrt{3}}{2} \sqrt{2 m_{I} 2 E_{F}(-\vec{q})} \int d^{3} p \frac{1}{4 \pi|\vec{p}|}\left(\hat{\phi}_{f_{1}^{\prime}, f_{2}}^{\left(M_{F}\left(1^{+}, S_{q q}=1\right)\right)}(|\vec{p}|)\right)^{*} \hat{\phi}_{f_{1}, f_{2}}^{\left(M_{I}\left(0^{-}\right)\right)}\left(\left|\vec{p}-\frac{m_{f_{2}}}{m_{f_{1}^{\prime}}+m_{f_{2}}}\right| \vec{q}|\vec{k}|\right) \\
& \sqrt{\frac{\widehat{E}_{f_{1}^{\prime}} \widehat{E}_{f_{1}}}{4 E_{f_{1}^{\prime}} E_{f_{1}}}}\left\{p_{z}\left(1-\frac{\left(-\frac{m_{f^{\prime}}}{m_{f_{1}^{\prime}}+m_{f_{2}}}|\vec{q}| \vec{k}-\vec{p}\right) \cdot\left(\frac{m_{f_{2}}}{m_{f_{1}^{\prime}}+m_{f_{2}}}|\vec{q}| \vec{k}-\vec{p}\right)}{\widehat{E}_{f_{1}^{\prime}} \widehat{E}_{f_{1}}}\right)\right. \\
& \left.+\frac{m_{f_{2}}-m_{f_{1}^{\prime}}}{m_{f_{1}^{\prime}}+m_{f_{2}}} \frac{p_{x}^{2}|\vec{q}|}{\widehat{E}_{f_{1}^{\prime}} \widehat{E}_{f 1}}\right\}
\end{aligned}
$$

- Case $J^{\pi}=2^{-}$

$$
\begin{aligned}
& V_{T \lambda=0}^{\left(2^{-}\right) 0}(|\vec{q}|)=i \sqrt{\frac{15}{2}} \sqrt{2 m_{I} 2 E_{F}(-\vec{q})} \int d^{3} p \frac{1}{4 \pi|\vec{p}|^{2}}\left(\hat{\phi}_{f_{1}^{\prime}, f_{2}}^{\left(M_{F}\left(2^{-}\right)\right)}(|\vec{p}|)\right)^{*} \hat{\phi}_{f_{1}, f_{2}}^{\left(M_{I}\left(0^{-}\right)\right)}\left(\left|\vec{p}-\frac{m_{f_{2}}}{m_{f_{1}^{\prime}}+m_{f_{2}}}\right| \vec{q}|\vec{k}|\right) \\
& \sqrt{\frac{\widehat{E}_{f_{1}^{\prime}} \widehat{E}_{f 1}}{4 E_{f_{1}^{\prime}} E_{f_{1}}}} \frac{p_{z}\left(p_{x}^{2}+p_{y}^{2}\right)|\vec{q}|}{\widehat{E}_{f_{1}^{\prime}} \widehat{E}_{f 1}} \\
& V_{T \lambda=+1}^{\left(2^{-}\right) 1}(|\vec{q}|)=i \frac{\sqrt{5}}{2} \sqrt{2 m_{l} 2 E_{F}(-\vec{q})} \int d^{3} p \frac{1}{4 \pi|\vec{p}|^{2}}\left(\hat{\phi}_{f_{1}^{\prime}, f_{2}}^{\left(M_{F}\left(2^{-}\right)\right)}(|\vec{p}|)\right)^{*} \hat{\phi}_{f_{1}, f_{2}}^{\left(M_{l}\left(0^{-}\right)\right)}\left(\left|\vec{p}-\frac{m_{f_{2}}}{m_{f_{1}^{\prime}}+m_{f_{2}}}\right| \vec{q}|\vec{k}|\right) \\
& \sqrt{\frac{\widehat{E}_{f_{1}^{\prime}} \widehat{E}_{f 1}}{4 E_{f_{1}^{\prime}} E_{f_{1}}}}\left\{\left(p_{z}^{2}-p_{x}^{2}\right)\left(\frac{-p_{z}-\frac{m_{f_{1}^{\prime}}}{m_{f_{1}^{\prime}}+m_{f_{2}}}|\vec{q}|}{\widehat{E}_{f_{1}^{\prime}}}-\frac{-p_{z}+\frac{m_{f_{2}}}{m_{f_{1}^{\prime}}}\left|\overrightarrow{m_{f_{2}}}\right|}{\widehat{E}_{f_{1}}}\right)\right. \\
& \left.-p_{z} p_{y}^{2}\left(\frac{1}{\widehat{E}_{f_{1}^{\prime}}}-\frac{1}{\widehat{E}_{f_{1}}}\right)\right\} \\
& V_{T \lambda=0}^{\left(2^{-}\right) 3}(|\vec{q}|)=i \sqrt{\frac{15}{2}} \sqrt{2 m_{I} 2 E_{F}(-\vec{q})} \int d^{3} p \frac{1}{4 \pi|\vec{p}|^{2}}\left(\hat{\phi}_{f_{1}^{\prime}, f_{2}}^{\left(M_{F}\left(2^{-}\right)\right)}(|\vec{p}|)\right)^{*} \hat{\phi}_{f_{1}, f_{2}}^{\left(M_{I}\left(0^{-}\right)\right)}\left(\left|\vec{p}-\frac{m_{f_{2}}}{m_{f_{1}^{\prime}}+m_{f_{2}}}\right| \vec{q}|\vec{k}|\right) \\
& \sqrt{\frac{\widehat{E}_{f_{1}^{\prime}} \widehat{E}_{f 1}}{4 E_{f_{1}^{\prime}} E_{f_{1}}}} p_{z}\left(p_{x}^{2}+p_{y}^{2}\right)\left(\frac{1}{\widehat{E}_{f_{1}^{\prime}}}-\frac{1}{\widehat{E}_{f_{1}}}\right)
\end{aligned}
$$




$$
\begin{aligned}
& A_{T \lambda=+1}^{\left(2^{-}\right) 1}(|\vec{q}|)=i \frac{\sqrt{5}}{2} \sqrt{2 m_{I} 2 E_{F}(-\vec{q})} \int d^{3} p \frac{1}{4 \pi|\vec{p}|^{2}}\left(\hat{\phi}_{f_{1}^{\prime}, f_{2}}^{\left(M_{F}\left(2^{-}\right)\right)}(|\vec{p}|)\right)^{*} \hat{\phi}_{f_{1}, f_{2}}^{\left(M_{I}\left(0^{-}\right)\right)}\left(\left|\vec{p}-\frac{m_{f_{2}}}{m_{f_{1}^{\prime}}+m_{f_{2}}}\right| \vec{q}|\vec{k}|\right) \\
& \sqrt{\frac{\widehat{E}_{f_{1}^{\prime}} \widehat{E}_{f 1}}{4 E_{f_{1}^{\prime}} E_{f_{1}}}}\left\{\left(p_{z}^{2}-p_{y}^{2}\right)\left(1-\frac{\left(-\frac{m_{f_{1}^{\prime}}}{m_{f_{1}^{\prime}}+m_{f_{2}}}|\vec{q}| \vec{k}-\vec{p}\right) \cdot\left(\frac{m_{f_{2}}}{m_{f_{1}^{\prime}}+m_{f_{2}}}|\vec{q}| \vec{k}-\vec{p}\right)}{\widehat{E}_{f_{1}^{\prime}} \widehat{E}_{f 1}}\right)\right. \\
& \left.-p_{z} p_{x}^{2}|\vec{q}| \frac{m_{f_{1}^{\prime}}-m_{f_{2}}}{m_{f_{1}^{\prime}}+m_{f_{2}}} \frac{1}{\widehat{E}_{f_{1}^{\prime}} \widehat{E}_{f_{1}}}\right\}
\end{aligned}
$$

- Case $J^{\pi}=2^{+}$

$$
\begin{aligned}
& V_{T \lambda=+1}^{\left(D_{s 2}^{*}\right) 1}(|\vec{q}|)=i \frac{\sqrt{3}}{2} \sqrt{2 m_{I} 2 E_{F}(-\vec{q})} \int d^{3} p \frac{1}{4 \pi|\vec{p}|}\left(\hat{\phi}_{f_{1}^{\prime}, f_{2}}^{\left(M_{F}\left(D_{s 2}^{*}\right)\right)}(|\vec{p}|)\right)^{*} \hat{\phi}_{f_{1}, f_{2}}^{\left(M_{I}\left(0^{-}\right)\right)}\left(\left|\vec{p}-\frac{m_{f_{2}}}{m_{f_{1}^{\prime}}+m_{f_{2}}}\right| \vec{q}|\vec{k}|\right) \\
& \sqrt{\frac{\widehat{E}_{f_{1}^{\prime}} \widehat{E}_{f 1}}{4 E_{f_{1}^{\prime}} E_{f_{1}}}}\left(\frac{p_{y}^{2}-p_{z}^{2}-p_{z}|\vec{q}| \frac{m_{f_{1}^{\prime}}}{m_{f_{1}^{\prime}}+m_{f_{2}}}}{\widehat{E}_{f_{1}^{\prime}}}-\frac{p_{y}^{2}-p_{z}^{2}+p_{z}|\vec{q}| \frac{m_{f_{2}}}{m_{f_{1}^{\prime}}+m_{f_{2}}}}{\widehat{E}_{f_{1}}}\right) \\
& A_{T \lambda=0}^{\left(D_{s 2}^{*}\right) 0}(|\vec{q}|)=\frac{-i}{\sqrt{2}} \sqrt{2 m_{I} 2 E_{F}(-\vec{q})} \int d^{3} p \frac{1}{4 \pi|\vec{p}|}\left(\hat{\phi}_{f_{1}^{\prime}, f_{2}}^{\left(M_{F}\left(D_{s 2}^{*}\right)\right)}(|\vec{p}|)\right)^{*} \hat{\phi}_{f_{1}, f_{2}}^{\left(M_{I}\left(0^{-}\right)\right)}\left(\left|\vec{p}-\frac{m_{f_{2}}}{m_{f_{1}^{\prime}}+m_{f_{2}}}\right| \vec{q}|\vec{k}|\right) \\
& \sqrt{\frac{\widehat{E}_{f_{1}^{\prime}} \widehat{E}_{f 1}}{4 E_{f_{1}^{\prime}} E_{f_{1}}}}\left(\frac{p_{x}^{2}+p_{y}^{2}-2 p_{z}^{2}-2 p_{z}|\vec{q}| \frac{m_{f_{1}^{\prime}}}{m_{f_{1}^{\prime}}+m_{f_{2}}}}{\widehat{E}_{f_{1}^{\prime}}}+\frac{p_{x}^{2}+p_{y}^{2}-2 p_{z}^{2}+2 p_{z}|\vec{q}| \frac{m_{f_{2}}}{m_{f_{1}^{\prime}}+m_{f_{2}}}}{\widehat{E}_{f_{1}}}\right) \\
& A_{T \lambda=+1}^{\left(D_{s 2}^{*}\right) 1}(|\vec{q}|)=i \frac{\sqrt{3}}{2} \sqrt{2 m_{I} 2 E_{F}(-\vec{q})} \int d^{3} p \frac{1}{4 \pi|\vec{p}|}\left(\hat{\phi}_{f_{1}^{\prime}, f_{2}}^{\left(M_{F_{2}}\left(D_{2}^{*}\right)\right)}(|\vec{p}|)\right)^{*} \hat{\phi}_{f_{1}, f_{2}}^{\left(M_{I}\left(0^{-}\right)\right)}\left(\left|\vec{p}-\frac{m_{f_{2}}}{m_{f_{1}^{\prime}}+m_{f_{2}}}\right| \vec{q}|\vec{k}|\right) \\
& \sqrt{\frac{\widehat{E}_{f_{1}^{\prime}} \widehat{E}_{f 1}}{4 E_{f_{1}^{\prime}} E_{f_{1}}}}\left\{p_{z}\left(1-\frac{\left(-\frac{m_{f_{1}^{\prime}}}{m_{f_{1}^{\prime}}+m_{f_{2}}}|\vec{q}| \vec{k}-\vec{p}\right) \cdot\left(\frac{m_{f_{2}}}{m_{f_{1}^{\prime}}+m_{f_{2}}}|\vec{q}| \vec{k}-\vec{p}\right)}{\widehat{E}_{f_{1}^{\prime}} \widehat{E}_{f 1}}\right)\right. \\
& \left.+\frac{4 p_{z} p_{x}^{2}-p_{x}^{2}|\vec{q}| \frac{m_{f_{2}}-m_{f_{1}^{\prime}}}{m_{f_{1}^{\prime}}+m_{f_{2}}}}{\widehat{E}_{f_{1}^{\prime}} \widehat{E}_{f 1}}\right\} \\
& A_{T \lambda=0}^{\left(D_{s 2}^{*}\right) 3}(|\vec{q}|)=-i \sqrt{2} \sqrt{2 m_{I} 2 E_{F}(-\vec{q})} \int d^{3} p \frac{1}{4 \pi|\vec{p}|}\left(\hat{\phi}_{f_{1}^{\prime}, f_{2}}^{\left(M_{F 2}\left(D_{s 2}^{*}\right)\right)}(|\vec{p}|)\right)^{*} \hat{\phi}_{f_{1}, f_{2}}^{\left(M_{I}\left(0^{-}\right)\right)}\left(\left|\vec{p}-\frac{m_{f_{2}}}{m_{f_{1}^{\prime}}+m_{f_{2}}}\right| \vec{q}|\vec{k}|\right) \\
& \sqrt{\frac{\widehat{E}_{f_{1}^{\prime}} \widehat{E}_{f 1}}{4 E_{f_{1}^{\prime}} E_{f_{1}}}}\left\{p_{z}\left(1-\frac{\left(-\frac{m_{f_{1}^{\prime}}}{m_{f_{1}^{\prime}}}\left|\overrightarrow{m_{f_{2}}}\right| \vec{q} \mid \vec{k}-\vec{p}\right) \cdot\left(\frac{m_{f_{2}}}{m_{f_{1}^{\prime}}+m_{f_{2}}}|\vec{q}| \vec{k}-\vec{p}\right)}{\widehat{E}_{f_{1}^{\prime}} \widehat{E}_{f 1}}\right)\right. \\
& +\frac{1}{\widehat{E}_{f_{1}^{\prime}} \widehat{E}_{f 1}}\left[2 p_{z}\left(-\frac{m_{f_{1}^{\prime}}}{m_{f_{1}^{\prime}}+m_{f_{2}}}|\vec{q}|-p_{z}\right) \cdot\left(\frac{m_{f_{2}}}{m_{f_{1}^{\prime}}+m_{f_{2}}}|\vec{q}|-p_{z}\right)\right. \\
& \left.\left.+\left(p_{x}^{2}+p_{y}^{2}\right)\left(-p_{z}+\frac{m_{f_{2}}-m_{f_{1}^{\prime}}}{2\left(m_{f_{1}^{\prime}}+m_{f_{2}}\right)}|\vec{q}|\right)\right]\right\}
\end{aligned}
$$

[1] R. Aaij et al. (LHCb collaboration), Phys.Rev. D87, 112009 (2013) arXiv:1304.6317 [hep-ex] 
[2] R. Aaij et al. (LHCb collaboration), Phys.Rev.Lett. (2013), arXiv:1304.6173 [hep-ex].

[3] D. Guadagnoli and G. Isidori, (2013), arXiv:1302.3909 [hep-ph].

[4] S. Blusk (LHCb Collaboration), (2012), arXiv:1212.4180 [hep-ex].

[5] R. Aaij et al. (LHCb collaboration), Phys.Rev. D87, 092007 (2013) arXiv:1302.5854 [hep-ex].

[6] R. Aaij et al. (The LHCb collaboration), (2013), arXiv:1308.4583 [hep-ex]

[7] R. Aaij et al. (LHCb collaboration), (2013), arXiv:1308.1428 [hep-ex]

[8] R. Aaij et al. (LHCb collaboration), Phys.Rev. D87, 071101 (2013) arXiv:1302.6446 [hep-ex].

[9] A. Dewhurst (ATLAS Collaboration), PoS BEAUTY2011, 013 (2011).

[10] G. Aad et al. (ATLAS Collaboration), JHEP 1212, 072 (2012), arXiv:1208.0572 [hep-ex]

[11] G. Giurgiu, PoS FPCP2010, 014 (2010), arXiv:1010.4082 [hep-ex]

[12] A. Abulencia et al. (CDF Collaboration), Phys.Rev.Lett. 96, 191801 (2006), arXiv:hep-ex/0508014 [hep-ex].

[13] L. Gong-Ru, L. Xin-Qiang, L. Yan-Min, and S. Fang, Acta Phys.Sin. 61, 241301 (2012)

[14] Y. Amhis (LHCb Collaboration), PoS HQL2012, 034 (2012), arXiv:1207.4639 [hep-ex]

[15] D. van Eijk (LHCb collaboration), (2011), arXiv:1109.4276 [hep-ex]

[16] T. Kuhr (Belle Collaboration, CDF Collaboration, D0 Collaboration), Conf.Proc. C100901, 84 (2010), arXiv:1103.0896 [hep-ex]

[17] G. Giurgiu (CDF Collaboration), PoS ICHEP2010, 236 (2010), arXiv:1012.0962 [hep-ex]

[18] B. Batell and M. Pospelov, Phys.Rev. D82, 054033 (2010) arXiv:1006.2127 [hep-ph]

[19] A. Abulencia et al. (CDF Collaboration), Phys.Rev.Lett. 97, 062003 (2006), arXiv:hep-ex/0606027 [hep-ex].

[20] N. Isgur and M. B. Wise, Phys.Lett. B232, 113 (1989)

[21] N. Isgur and M. B. Wise, Phys.Lett. B237, 527 (1990)

[22] H. Georgi, Phys.Lett. B240, 447 (1990),

[23] C. Albertus, E. Hernandez, J. Nieves, and J. Verde-Velasco, Phys.Rev. D71, 113006 (2005) arXiv:hep-ph/0502219 [hep-ph].

[24] E. Hernandez, J. Nieves, and J. Verde-Velasco, Eur.Phys.J. A31, 714 (2007) arXiv:hep-ph/0610125 [hep-ph]

[25] C. Albertus, E. Hernandez, J. Nieves, and J. Verde-Velasco, Eur.Phys.J. A32, 183 (2007) arXiv:hep-ph/0610030 [hep-ph]

[26] C. Albertus, E. Hernandez, and J. Nieves, Phys.Rev. D85, 094035 (2012) arXiv:1202.4861 [hep-ph]

[27] C. Albertus, E. Hernandez, and J. Nieves, Phys.Lett. B704, 499 (2011) arXiv:1108.1296 [hep-ph]

[28] R. Faustov and V. Galkin, Phys.Rev. D87, 034033 (2013) arXiv:1212.3167

[29] R. Faustov and V. Galkin, Phys.Rev. D87, 094028 (2013) arXiv:1304.3255

[30] J. Sun, Z. Xiong, Y. Yang, and G. Lu, Eur.Phys.J. C73, 2437 (2013) arXiv:1305.0691 [hep-ph]

[31] X. Yu, Z.-T. Zou, and C.-D. Lu, (2013), arXiv:1307.7485 [hep-ph]

[32] X. J. Chen, H. F. Fu, and G. L. Wang, J.Phys. G 39, 045002.

[33] R.-H. Li, C.-D. Lu, and Y.-M. Wang, Phys.Rev. D80, 014005 (2009) arXiv:0905.3259 [hep-ph]

[34] K. Azizi, Nucl. Phys. B 801, 70 (2008).

[35] K. Azizi and M. Bayar, Phys.Rev. D78, 054011 (2008) arXiv:0806.0578 [hep-ph]

[36] P. Blasi, P. Colangelo, G. Nardulli, and N. Paver,Phys.Rev. D49, 238 (1994) arXiv:hep-ph/9307290 [hep-ph]

[37] S.-M. Zhao, X. Liu, and S.-J. Li, Eur.Phys.J. C51, 601 (2007) arXiv:hep-ph/0612008 [hep-ph].

[38] M. A. Ivanov, J. G. Korner, and P. Santorelli, Phys.Rev. D71, 094006 (2005), arXiv:hep-ph/0501051 [hep-ph].

[39] J. Beringer et al. (Particle Data Group), Phys. Rev. D 86, 010001 (2012)

[40] E. Hernandez, J. Nieves, and J. Verde-Velasco, Phys.Rev. D74, 074008 (2006) arXiv:hep-ph/0607150 [hep-ph]

[41] R. Bhaduri, L. Cohler, and Y. Nogami, Nuovo Cim. A65, 376 (1981)

[42] B. Silvestre-Brac, Few Body Syst. 20, 1 (1996)

[43] J. Korner and G. Schuler, Z.Phys. C46, 93 (1990)

[44] J. Segovia, C. Albertus, D. Entem, F. Fernandez, E. Hernandez, et al., Phys.Rev. D84, 094029 (2011), arXiv:1107.4248 [hep-ph]

[45] J. Vijande, F. Fernandez, and A. Valcarce, J.Phys. G31, 481 (2005), arXiv:hep-ph/0411299 [hep-ph]

[46] D. Ebert, R. Faustov, and V. Galkin, Phys.Rev. D75, 074008 (2007), arXiv:hep-ph/0611307 [hep-ph]

[47] M. Beneke, G. Buchalla, M. Neubert, and C. T. Sachrajda, Phys.Rev.Lett. 83, 1914 (1999) arXiv:hep-ph/9905312 [hep-ph]

[48] M. A. Ivanov, J. G. Korner, and P. Santorelli, Phys.Rev. D73, 054024 (2006), arXiv:hep-ph/0602050 [hep-ph].

[49] K. Azizi, R. Khosravi, and F. Falahati, Int.J.Mod.Phys. A24, 5845 (2009) arXiv:0811.2671 [hep-ph]

[50] R.-H. Li, C.-D. Lu, and H. Zou, Phys.Rev. D78, 014018 (2008) arXiv:0803.1073 [hep-ph]

[51] M. A. Ivanov, J. G. Korner, S. G. Kovalenko, P. Santorelli, and G. G. Saidullaeva, Phys.Rev. D85, 034004 (2012). arXiv:1112.3536 [hep-ph] 